\title{
ABSOLUTELY STABLE LOCAL DISCONTINUOUS GALERKIN METHODS FOR THE HELMHOLTZ EQUATION WITH LARGE WAVE NUMBER
}

\author{
XIAOBING FENG AND YULONG XING
}

\begin{abstract}
This paper develops and analyzes two local discontinuous Galerkin (LDG) methods using piecewise linear polynomials for the Helmholtz equation with the first order absorbing boundary condition in the high frequency regime. It is shown that the proposed LDG methods are stable for all positive wave number $k$ and all positive mesh size $h$. Energy norm and $L^{2}$-norm error estimates are derived for both LDG methods in all mesh parameter regimes including pre-asymptotic regime (i.e., $k^{2} h \gtrsim 1$ ). To analyze the proposed LDG methods, they are recast and treated as (nonconforming) mixed finite element methods. The crux of the analysis is to show that the sesquilinear form associated with each LDG method satisfies a coercivity property in all mesh parameter regimes. These coercivity properties then easily infer the desired discrete stability estimates for the solutions of the proposed LDG methods. In return, the discrete stabilities not only guarantee the well-posedness of the LDG methods but also play a crucial role in the error analysis. Numerical experiments are also presented in the paper to validate the theoretical results and to compare the performance of the proposed two LDG methods.
\end{abstract}

\section{INTRODUCTION}

This paper is the third installment in a series [10, 11] which is devoted to developing absolutely stable discontinuous Galerkin (DG) methods for the following prototypical Helmholtz problem with large wave number:

$$
\begin{aligned}
-\Delta u-k^{2} u=f & \text { in } \Omega \subset \mathbf{R}^{d}, d=2,3, \\
\frac{\partial u}{\partial \mathbf{n}_{\Omega}}+\mathbf{i} k u=g & \text { on } \Gamma=\partial \Omega,
\end{aligned}
$$

where $\mathbf{i}=\sqrt{-1}$ denotes the imaginary unit. $k \in \mathbf{R}_{+}$is a given positive (large) number and known as the wave number. (1.2) is the so-called first order absorbing boundary condition 9 .

We recall that 10, 11 focused on designing and analyzing $h$ - and $h p$-interior penalty discontinuous Galerkin (IPDG) methods which are absolutely stable (with

Received by the editor October 16, 2010 and, in revised form, August 25, 2011 and November $10,2011$.

2010 Mathematics Subject Classification. Primary 65N12, 65N15, 65N30, 78A40 .

Key words and phrases. Helmholtz equation, time harmonic waves, local discontinuous Galerkin methods, stability, error estimates.

The work of the first author was partially supported by the NSF grants DMS-0710831 and DMS-1016173. The research of the second author was partially sponsored by the Office of Advanced Scientific Computing Research; U.S. Department of Energy. The work of the second author was performed at the ORNL, which is managed by UT-Battelle, LLC under Contract No. DE-AC05-00OR22725. 
respect to wave number $k$ and mesh size $h$ ) and optimally convergent (with respect to $h$ ). The main ideas of [10, 11] are to introduce some novel interior penalty terms in the sesquilinear forms of the proposed IPDG methods and to use a non-standard analytical tool, which is based on a Rellich identity technique, to prove the desired stability and error estimates. The numerical experiment results show that the absolutely stable IPDG methods significantly outperform the standard finite element and finite difference methods, which are known only to be stable under stringent mesh constraints $h k \lesssim 1$ or $h k^{2} \lesssim 1$ (cf. [8, 17]), for the Helmholtz problem. Moreover, the numerical experiment results also show that these IPDG methods are capable to correctly track the phases of the highly oscillatory waves even when the mesh violates the "rule-of-thumb" condition (i.e., 6 - 10 grid points must be used in a wave length). The main difficulty of analyzing the Helmholtz type problems is caused by the strong indefiniteness of the Helmholtz equation which in turn makes it hard to establish stability estimates for its numerical approximations. The loss of stability in the case of large wave numbers results in an additional pollution error (besides the interpolation error) in the global error bounds. Extensive research has been done to address the question of whether it is possible to reduce the pollution effect; we refer the reader to Chapter 4 of [16] and the references therein for an detailed exposition in this direction.

Motivated by the success of [10, 11], the primary objective of this paper is to extend the work of [10, 11] to the local discontinuous Galerkin (LDG) formulation, which is known to be more "physical" and flexible than the IPDG formulation on designing DG schemes [1, 6]. As it is well known now, the key step for constructing LDG methods is to design the numerical fluxes. As soon as the numerical fluxes are selected, for a large class of coercive elliptic and parabolic second order problems, there is a general framework for carrying out convergence analysis of LDG methods [1. Unfortunately, this general framework does not apply to the Helmholtz type problems which is extremely noncoercive/indefinite for large wave number $k$. Nevertheless, when designing the numerical fluxes for our LDG methods, we borrow the idea of [1 by establishing connections between our LDG methods and the IPDG methods of [10, 11] although it turns out that the IPDG methods of [10, 11] do not have exactly equivalent LDG formulations due to the non-standard penalty terms used in [10, 11]. This then leads to the construction of our first LDG method. It is proved and numerically verified that this LDG method is absolutely stable and optimally convergent for the scalar variable. However, it is sub-optimal for the vector/flux variable. To improve the approximation accuracy for the vector/flux variable, we design another set of numerical fluxes which result in the construction of our second LDG method. It is proved that the second LDG method is also absolutely stable and gives a better approximation for the vector/flux variable than the first method. On the other hand, it is computationally more expensive than the first LDG method, which is expected.

To analyze the proposed LDG methods, we take an opposite approach to that advocated in [1, that is, instead of converting LDG methods to their "equivalent" IPDG methods in the primal form, we recast and treat our LDG methods as nonconforming mixed finite element methods. To avoid using the standard techniques such as Schatz' argument (cf. [2, 8]) or Babuška's inf-sup condition argument [1] to derive error estimates (and to prove stability), both approaches would lead to stringent mesh constraints, our main idea is to prove some (generalized) coercivity 
properties, which hold without any mesh constraint, for both LDG methods. These coercivity properties then immediately infer the desired discrete stability estimates for the solutions of the proposed LDG methods. In return, the stability results not only guarantee the well-posedness of the LDG methods in all mesh parameter regimes but also play a crucial role in our error analysis.

It should be pointed out that a lot of work has recently been done on developing DG methods using piecewise plane wave functions, opposed to simpler piecewise polynomial functions as done in this paper, for the Helmholtz type problems. However, to the best of our knowledge, none of these plane wave DG methods are proved to be absolutely stable with respect to wave number $k$ and mesh size $h$. We refer the reader to [13, 15, 18, and the references therein for more discussions in this direction. We also refer to [10, 11] for more discussions and references on other discretization techniques for the Helmholtz type problems.

This paper consists of four additional sections. In Section 2, we introduce the notation used in this paper and present the derivations of our two LDG methods. In Section 3, we present a detailed stability analysis for both LDG methods. The main task of the section is to prove a coercivity property for each proposed LDG method. In Section 4 a nonstandard two-step error estimate procedure is used to derive error estimates for the proposed LDG methods. Once again, the stability estimates established in Section 3 play a crucial role. Finally, Section 5 contains some numerical experiments which are designed to verify the theoretical error bounds proved in Section 4 and to compare the performance of the proposed two LDG methods.

\section{Formulation of LOCAL Discontinuous Galerkin methods}

The standard space, norm and inner product notation, are adopted in this paper. Their definitions can be found in [1, 2, 5, 19. In particular, $(\cdot, \cdot)_{Q}$ and $\langle\cdot, \cdot\rangle_{\Sigma}$ for $\Sigma \subset \partial Q$ denote the $L^{2}$-inner product on complex-valued $L^{2}(Q)$ and $L^{2}(\Sigma)$ spaces, respectively. $(\cdot, \cdot):=(\cdot, \cdot)_{\Omega}$ and $\langle\cdot, \cdot\rangle:=\langle\cdot, \cdot\rangle_{\partial \Omega}$. Throughout the paper, $C$ is used to denote a generic positive constant which is independent of $h$ and $k$. We also use the shorthand notation $A \lesssim B$ and $B \gtrsim A$ for the inequality $A \leq C B$ and $B \geq C A$. $A \simeq B$ is a shorthand notation for the statement $A \lesssim B$ and $B \lesssim A$.

Assume that $\Omega \subset \mathbf{R}^{d}(d=2,3)$ is a bounded and strictly star-shaped domain with respect to a point $x_{\Omega} \in \Omega$. We now recall the definition of star-shaped domains.

Definition 2.1. $Q \subset \mathbf{R}^{d}$ is said to be a star-shaped domain with respect to $x_{Q} \in Q$ if there exists a nonnegative constant $c_{Q}$ such that

$$
\left(x-x_{Q}\right) \cdot \mathbf{n}_{Q} \geq c_{Q} \quad \forall x \in \partial Q .
$$

$Q \subset \mathbf{R}^{d}$ is said to be strictly star-shaped if $c_{Q}$ is positive.

Let $\mathcal{T}_{h}$ be a family of partitions of $\Omega$ parameterized by $h>0$. For any triangle/tetrahedron $K \in \mathcal{T}_{h}$, we define $h_{K}:=\operatorname{diam}(K)$ and $h:=\max _{K \in \mathcal{T}_{h}} h_{K}$. Similarly, for each edge/face $e$ of $K \in \mathcal{T}_{h}$, define $h_{e}:=\operatorname{diam}(e)$. We assume that the elements of $\mathcal{T}_{h}$ satisfy the minimal angle condition. We also define

$$
\begin{aligned}
\mathcal{E}_{h}^{I} & :=\text { set of all interior edges } / \text { faces of } \mathcal{T}_{h}, \\
\mathcal{E}_{h}^{B} & :=\text { set of all boundary edges/faces of } \mathcal{T}_{h} \text { on } \Gamma=\partial \Omega, \\
\mathcal{E}_{h} & :=\mathcal{E}_{h}^{I} \cup \mathcal{E}_{h}^{B} .
\end{aligned}
$$


Let $e$ be an interior edge shared by two elements $K_{1}$ and $K_{2}$ whose unit outward normal vectors are denoted by $\mathbf{n}_{1}$ and $\mathbf{n}_{2}$. For a scalar function $v$, let $v_{i}=\left.v\right|_{\partial K_{i}}$, and define

$$
\{v\}=\frac{1}{2}\left(v_{1}+v_{2}\right), \quad[v]=v_{K_{1}}-v_{K_{2}}, \quad[[v]]=v_{1} \mathbf{n}_{1}+v_{2} \mathbf{n}_{2} \quad \text { on } e \in \mathcal{E}_{h}^{I},
$$

For a vector field $\mathbf{v}$, let $\mathbf{v}_{i}=\left.\mathbf{v}\right|_{\partial K_{i}}$ and define

$$
\{\mathbf{v}\}=\frac{1}{2}\left(\mathbf{v}_{1}+\mathbf{v}_{2}\right), \quad[\mathbf{v}]=\mathbf{v}_{K_{1}}-\mathbf{v}_{K_{2}}, \quad[[\mathbf{v}]]=\mathbf{v}_{1} \cdot \mathbf{n}_{1}+\mathbf{v}_{2} \cdot \mathbf{n}_{2} \quad \text { on } e \in \mathcal{E}_{h}^{I} .
$$

It is well known now (cf. [1]) that the first step for formulating an LDG method is to rewrite the given PDE as a first order system by introducing an auxiliary variable. For the Helmholtz problem (1.1)-(1.2) we have

$$
\begin{aligned}
\boldsymbol{\sigma} & =\nabla u & & \text { in } \Omega, \\
-\operatorname{div} \boldsymbol{\sigma}-k^{2} u & =f & & \text { in } \Omega, \\
\frac{\partial u}{\partial \mathbf{n}_{\Omega}}+\mathbf{i} k u & =g & & \text { on } \Gamma .
\end{aligned}
$$

Clearly, the vector-valued function (often called the flux variable) $\boldsymbol{\sigma}$ is the auxiliary variable.

Then, multiplying (2.2) and (2.3) by test functions $\bar{\tau}$ and $\bar{v}$, respectively, and integrating both equations over an element $K \in \mathcal{T}_{h}$ yields

$$
\begin{aligned}
\int_{K} \boldsymbol{\sigma} \cdot \overline{\boldsymbol{\tau}} d x & =-\int_{K} u \operatorname{div} \overline{\boldsymbol{\tau}} d x+\int_{\partial K} u \mathbf{n}_{K} \cdot \overline{\boldsymbol{\tau}} d s, \\
\int_{K} \boldsymbol{\sigma} \cdot \nabla \bar{v} d x-k^{2} \int_{K} u \bar{v} d x & =\int_{K} f \bar{v} d x+\int_{\partial K} \boldsymbol{\sigma} \cdot \mathbf{n}_{K} \bar{v} d s,
\end{aligned}
$$

where $\mathbf{n}_{K}$ denotes the unit outward normal vector to $\partial K$. The above equations form the weak formulation one uses to define LDG methods for the Helmholtz problem (1.1)-(1.2).

Next, we define LDG spaces as follows:

$$
\begin{aligned}
V_{h} & :=\left\{v \in L^{2}(\Omega) ;\left.\operatorname{Re}(v)\right|_{K},\left.\operatorname{Im}(v)\right|_{K} \in P_{r}(K) \forall K \in \mathcal{T}_{h}\right\}, \\
\boldsymbol{\Sigma}_{h} & :=\left\{\boldsymbol{\tau} \in\left(L^{2}(\Omega)\right)^{d} ;\left.\operatorname{Re}(\boldsymbol{\tau})\right|_{K},\left.\operatorname{Im}(\boldsymbol{\tau})\right|_{K} \in\left(P_{\ell}(K)\right)^{d} \forall K \in \mathcal{T}_{h}\right\},
\end{aligned}
$$

where $P_{r}(K)(r \geq 1)$ stands for the set of all polynomials of degree less than or equal to $r$ on $K$.

Finally, we are ready to define the following general LDG formulation: Find $\left(u_{h}, \boldsymbol{\sigma}_{h}\right) \in V_{h} \times \boldsymbol{\Sigma}_{h}$ such that for all $K \in \Gamma_{h}$ it holds that

$$
\begin{aligned}
\int_{K} \boldsymbol{\sigma}_{h} \cdot \overline{\boldsymbol{\tau}}_{h} d x & =-\int_{K} u_{h} \operatorname{div} \overline{\boldsymbol{\tau}}_{h} d x+\int_{\partial K} \hat{u}_{K} \mathbf{n}_{K} \cdot \overline{\boldsymbol{\tau}}_{h} d s, \\
\int_{K} \boldsymbol{\sigma}_{h} \cdot \nabla \bar{v}_{h} d x-k^{2} \int_{K} u_{h} \bar{v}_{h} d x & =\int_{K} f \bar{v}_{h} d x+\int_{\partial K} \hat{\boldsymbol{\sigma}}_{K} \cdot \mathbf{n}_{K} \bar{v}_{h} d s
\end{aligned}
$$

for any $\left(v_{h}, \boldsymbol{\tau}_{h}\right) \in V_{h} \times \boldsymbol{\Sigma}_{h}$, where the quantities $\hat{u}_{K}$ and $\hat{\boldsymbol{\sigma}}_{K}$, which are called numerical fluxes, are, respectively, approximations to $\sigma=\nabla u$ and $u$ on the boundary $\partial K$ of $K$. As it is well-known now the most important issue for all LDG methods is how to choose the numerical fluxes. The different choices of the numerical fluxes obviously lead to different LDG methods. It is easy to understand that these numerical fluxes must be chosen carefully in order to ensure the stability and accuracy of the resulted LDG methods. 
In this paper, we shall only consider the linear element case (i.e., $r=\ell=1$ ) and propose two sets of numerical fluxes $\left(\hat{u}_{K}, \hat{\boldsymbol{\sigma}}_{K}\right)$, which lead to two LDG methods. Our choices of numerical fluxes are inspired by the interior penalty discontinuous Galerkin (IPDG) methods of [10] and are identified with the help of the unified DG framework of [1] which bridges the primal DG formulations (e.g., IPDG methods) and the flux DG formulations (i.e., LDG methods).

(1) LDG method \#1: Set

$$
\begin{array}{lll}
\hat{\boldsymbol{\sigma}}_{K}=\left\{\nabla_{h} u_{h}\right\}-\mathbf{i} \beta\left[\left[u_{h}\right]\right], & \left.\hat{u}_{K}=\left\{u_{h}\right\}+\mathbf{i} \delta\left[\left[\nabla_{h} u_{h}\right]\right]\right] & \text { on } e \in \mathcal{E}_{h}^{I}, \\
\hat{\boldsymbol{\sigma}}_{K}=-\mathbf{i} k u_{h} \mathbf{n}_{K}+g \mathbf{n}_{K}, & \hat{u}_{K}=u_{h} & \text { on } e \in \mathcal{E}_{h}^{B} ;
\end{array}
$$

(2) LDG method \#2: Set

$$
\begin{array}{lll}
\hat{\boldsymbol{\sigma}}_{K}=\left\{\boldsymbol{\sigma}_{h}\right\}-\mathbf{i} \beta\left[\left[u_{h}\right]\right], & \hat{u}_{K}=\left\{u_{h}\right\}+\mathbf{i} \delta\left[\left[\boldsymbol{\sigma}_{h}\right]\right] & \text { on } e \in \mathcal{E}_{h}^{I}, \\
\hat{\boldsymbol{\sigma}}_{K}=-\mathbf{i} k u_{h} \mathbf{n}_{K}+g \mathbf{n}_{K}, & \hat{u}_{K}=u_{h} & \text { on } e \in \mathcal{E}_{h}^{B},
\end{array}
$$

where $\beta$ and $\delta$ are positive constants to be specified later and $\nabla_{h}$ denotes the piecewisely defined gradient operator over $\mathcal{T}_{h}$, that is, $\left.\nabla_{h}\right|_{K}=\left.\nabla\right|_{K} \forall K \in \mathcal{T}_{h}$.

For the reader's convenience, we now briefly sketch the derivation of the primal DG formulation corresponding to our LDG method \#1 by adapting the derivation given in the general framework of [1].

Substituting the numerical fluxes of LDG method \#1 into (2.7) and (2.8), summing the resulting equations over all elements $K \in \mathcal{T}_{h}$ and using the following integration by parts identity

$$
\begin{aligned}
\left(u_{h}, \operatorname{div}_{h} \boldsymbol{\tau}_{h}\right)_{\Omega}=-\left(\nabla_{h} u_{h}, \boldsymbol{\tau}_{h}\right)_{\Omega} & +\sum_{e \in \mathcal{E}_{h}^{I}}\left(\left\langle\left\{u_{h}\right\},\left[\left[\boldsymbol{\tau}_{h}\right]\right]\right\rangle_{e}\right. \\
& \left.+\left\langle\left[\left[u_{h}\right]\right],\left\{\boldsymbol{\tau}_{h}\right\}\right\rangle_{e}\right)+\left\langle u_{h}, \mathbf{n}_{K} \cdot \boldsymbol{\tau}_{h}\right\rangle_{\Gamma},
\end{aligned}
$$

we get

$$
\begin{aligned}
&\left(\boldsymbol{\sigma}_{h}, \boldsymbol{\tau}_{h}\right)_{\Omega}-\left(\nabla_{h} u_{h}, \boldsymbol{\tau}_{h}\right)_{\Omega} \\
&-\sum_{e \in \mathcal{E}_{h}^{I}}\left(\mathbf{i} \delta\left\langle\left[\left[\nabla_{h} u_{h}\right]\right],\left[\left[\boldsymbol{\tau}_{h}\right]\right]\right\rangle_{e}-\left\langle\left[\left[u_{h}\right]\right],\left\{\boldsymbol{\tau}_{h}\right\}\right\rangle_{e}\right)=0, \\
&\left(\boldsymbol{\sigma}_{h}, \nabla_{h} v_{h}\right)_{\Omega}-k^{2}\left(u_{h}, v_{h}\right)_{\Omega}-\sum_{e \in \mathcal{E}_{h}^{I}}\left\langle\left\{\nabla_{h} u_{h}\right\}-\mathbf{i} \beta\left[\left[u_{h}\right]\right],\left[\left[v_{h}\right]\right]\right\rangle_{e} \\
&+\mathbf{i} k\left\langle u_{h}, v_{h}\right\rangle_{\Gamma}=\left(f, v_{h}\right)_{\Omega}+\left\langle g, v_{h}\right\rangle_{\Gamma} .
\end{aligned}
$$

Setting $\boldsymbol{\tau}=\nabla_{h} v_{h}$ in (2.9) and subtracting the resulting equation from (2.10) then leads to the formulation

$$
\mathcal{A}_{h}\left(u_{h}, v_{h}\right)-k^{2}\left(u_{h}, v_{h}\right)_{\Omega}=F\left(v_{h}\right) \quad \forall v_{h} \in V_{h},
$$


where

$$
\begin{aligned}
& \mathcal{A}_{h}\left(u_{h}, v_{h}\right):=\left(\nabla_{h} u_{h}, \nabla_{h} v_{h}\right)_{\Omega}+\mathbf{i} k\left\langle u_{h}, v_{h}\right\rangle_{\Gamma} \\
&+\sum_{e \in \mathcal{E}_{h}^{I}} \mathbf{i}\left(\delta\left\langle\left[\left[\nabla_{h} u_{h}\right]\right],\left[\left[\nabla_{h} v_{h}\right]\right]\right\rangle_{e}+\beta\left\langle\left[\left[u_{h}\right]\right],\left[\left[v_{h}\right]\right]\right\rangle_{e}\right) \\
&-\sum_{e \in \mathcal{E}_{h}^{I}}\left(\left\langle\left[\left[u_{h}\right]\right],\left\{\nabla_{h} v_{h}\right\}\right\rangle_{e}+\left\langle\left\{\nabla_{h} u_{h}\right\},\left[\left[v_{h}\right]\right]\right\rangle_{e}\right) . \\
& F\left(v_{h}\right):=\left(f, v_{h}\right)_{\Omega}+\left\langle g, v_{h}\right\rangle_{\Gamma} .
\end{aligned}
$$

Hence, (2.11) is the corresponding primal (i.e., IPDG) formulation of our LDG method \#1. Comparing (2.11) with the IPDG formulation of [10], we see that all the interior penalty terms of (2.11) also appear in the IPDG formulation of [10. In fact, it is exactly by reversing the order of the above derivation that leads to the discovery of the numerical fluxes of LDG method \#1.

Remark 2.1. We have used the terminology "LDG" liberally in this paper. Strictly speaking, the two LDG methods we proposed above are not "LDG" methods, instead, it can be proved that both methods actually are hybridizable LDG (or LDGH) methods by the definition of [4].

\section{Stability ANALYSis}

From their constructions, it is easy to see that both LDG methods proposed in the previous section are consistent schemes for the Helmholtz problem (1.1)-(1.2). For coercive elliptic (and parabolic) problems, the stability of such a numerical scheme can be proved easily as demonstrated in [1] (the same statement is true for their corresponding PDE stability analysis). However, the Helmholtz problem (1.1) - (1.2) is an indefinite problem and it becomes notoriously noncoercive for large wave number $k$. Deriving its stability estimates (i.e., a priori estimates of its PDE solution), particularly wave-number-dependent estimates, has been proved not to be an easy job (cf. [7, 14 and the references therein). Numerically, such a quest has been known to be even harder because of the lower order of the smoothness and the inflexibility of (piecewise) approximation functions (cf. [16, 17] and the references therein). The stability of numerical methods in all the above quoted references was proved under some restrictive mesh constraints. An open question was then raised by Zienkiewicz [20] which asks whether it is possible to construct an absolutely stable numerical method in the $L^{2}$-norm (i.e., the $L^{2}$-norms of numerical solutions are bounded without restriction on the mesh size $h$, but the bound may depend on $k$ and $h$ ) for the Helmholtz equation. The first such methods were recently developed in 10, 11, which happen to belong to the IPDG family, for the Helmholtz problem (1.1) (1.2).

The goal of this section is to show that in the case of the linear element (i.e., $r=$ $\ell=1$ ) the LDG methods \#1 and \#2 proposed in the previous section have similar stability properties to the IPDG methods of [10, 11, that is, the LDG methods \#1 and \#2 are absolutely stable for all mesh size $h>0$ and all wave numbers $k>0$. To establish this result, unlike the approach used and advocated in the general framework of [1] which converts an LDG method into its equivalent primal method and then analyzes the latter using the standard finite element Galerkin techniques, we shall directly fit the LDG methods \#1 and \#2 into the (nonconforming) mixed 
method framework as done in [17 and adapt the mixed method techniques to prove the desired stability result. It is well known that the key ingredient for the mixed method approach is to establish the inf-sup or Babuška-Brezzi condition for the (augmented) sesquilinear forms for each method. However, we are not able to prove such an inf-sup condition for either method without imposing stringent mesh constraints. To overcome the difficulty, our main idea is to prove some generalized coercivity properties, which hold for all $h, k>0$ for both LDG methods and for general domains including nonstar-shaped ones. All of these are possible because of the special design of the sesquilinear forms and the special property $\Delta v_{h}=0$ in $K \in$ $\mathcal{T}_{h}$ for all piecewise linear functions $v_{h}$. It turns out that these coercivity properties are sufficient to infer the desired discrete stability estimates for the solutions of the LDG methods \#1 and \#2.

3.1. Absolute stability of LDG method \#1. We first recast the LDG method \#1 as the following nonconforming mixed method, which is easily obtained by adding (2.9) and (2.10). Find $\left(u_{h}, \boldsymbol{\sigma}_{h}\right) \in V_{h} \times \boldsymbol{\Sigma}_{h}$ such that

$$
A_{h}\left(u_{h}, \boldsymbol{\sigma}_{h} ; v_{h}, \boldsymbol{\tau}_{h}\right)=F\left(v_{h}, \boldsymbol{\tau}_{h}\right) \quad \forall\left(v_{h}, \boldsymbol{\tau}_{h}\right) \in V_{h} \times \boldsymbol{\Sigma}_{h},
$$

where

$$
\begin{aligned}
& A_{h}\left(w_{h}, \boldsymbol{\chi}_{h} ; v_{h}, \boldsymbol{\tau}_{h}\right)=\left(\boldsymbol{\chi}_{h}, \nabla_{h} v_{h}\right)_{\Omega}-k^{2}\left(w_{h}, v_{h}\right)_{\Omega}+\mathbf{i} k\left\langle w_{h}, v_{h}\right\rangle_{\Gamma} \\
&-\sum_{e \in \mathcal{E}_{h}^{I}}\left\langle\left\{\nabla_{h} w_{h}\right\}-\mathbf{i} \beta\left[\left[w_{h}\right]\right],\left[\left[v_{h}\right]\right]\right\rangle_{e} \\
&-\sum_{e \in \mathcal{E}_{h}^{I}}\left(\mathbf{i} \delta\left\langle\left[\left[\nabla_{h} w_{h}\right]\right],\left[\left[\boldsymbol{\tau}_{h}\right]\right]\right\rangle_{e}-\left\langle\left[\left[w_{h}\right]\right],\left\{\boldsymbol{\tau}_{h}\right\}\right\rangle_{e}\right) \\
&+\left(\chi_{h}, \boldsymbol{\tau}_{h}\right)_{\Omega}-\left(\nabla_{h} w_{h}, \boldsymbol{\tau}_{h}\right)_{\Omega} . \\
& F\left(v_{h}, \boldsymbol{\tau}_{h}\right):=\left(f, v_{h}\right)_{\Omega}+\left\langle g, v_{h}\right\rangle_{\Gamma} .
\end{aligned}
$$

3.1.1. Weak coercivity of $A_{h}$. The goal of this subsection is to show that the sesquilinear form $A_{h}$ defined in (3.2) satisfies an inf-sup condition, which will play a vital role for us to establish the absolute stability of the LDG method \#1 in the next subsection.

Proposition 3.1. There exists an $h$-and $k$-independent constant $c_{1}>0$ such that for any $\left(w_{h}, \chi_{h}\right) \in V_{h} \times \boldsymbol{\Sigma}_{h}$,

$$
\sup _{\substack{\left(v_{h}, \tau_{h}\right) \in V_{h} \times \Sigma_{h} \\ v_{h} \neq 0}} \frac{\left|A_{h}\left(w_{h}, \boldsymbol{\chi}_{h} ; v_{h}, \boldsymbol{\tau}_{h}\right)\right|}{\left\|v_{h}\right\|_{D G}} \geq \frac{c_{1}}{\gamma_{1}}\left\|w_{h}\right\|_{D G},
$$

where

$$
\begin{aligned}
& \gamma_{1}:=1+\max _{e \in \mathcal{E}_{h}^{B}} \frac{1}{k h_{e}}+\max _{e \in \mathcal{E}_{h}^{I}}\left(\frac{1}{\beta h_{e}}+\frac{1}{k^{2} \delta h_{e}}\right), \\
& \left\|w_{h}\right\|_{D G}:=\left(k^{2}\left\|w_{h}\right\|_{L^{2}(\Omega)}^{2}+k\left\|w_{h}\right\|_{L^{2}(\Gamma)}^{2}+\left|w_{h}\right|_{1, h}^{2}\right)^{\frac{1}{2}}, \\
& \left|w_{h}\right|_{1, h}:=\left(\sum_{K \in \mathcal{T}_{h}}\left\|\nabla w_{h}\right\|_{L^{2}(K)}^{2}\right)^{\frac{1}{2}} .
\end{aligned}
$$

Proof. Since the proof is long, we divide the process into threes steps. 
Step 1. Taking the test function. We first choose the test function $\left(v_{h}, \boldsymbol{\tau}_{h}\right)=$ $\left(w_{h},-\nabla_{h} w_{h}\right)$ to get

$$
\begin{aligned}
A_{h}\left(w_{h}, \chi_{h} ; v_{h}, \boldsymbol{\tau}_{h}\right)= & A_{h}\left(w_{h}, \chi_{h} ; w_{h},-\nabla_{h} w_{h}\right) \\
= & \left(\nabla_{h} w_{h}, \nabla_{h} w_{h}\right)_{\Omega}-k^{2}\left(w_{h}, w_{h}\right)_{\Omega}+\mathbf{i} k\left\langle w_{h}, w_{h}\right\rangle_{\Gamma} \\
& \quad+\sum_{e \in \mathcal{E}_{h}^{I}}\left(\mathbf{i} \delta\left\langle\left[\left[\nabla_{h} w_{h}\right]\right],\left[\left[\nabla_{h} w_{h}\right]\right]\right\rangle_{e}-\left\langle\left[\left[w_{h}\right]\right],\left\{\nabla_{h} w_{h}\right\}\right\rangle_{e}\right) \\
& -\sum_{e \in \mathcal{E}_{h}^{I}}\left\langle\left\{\nabla_{h} w_{h}\right\}-\mathbf{i} \beta\left[\left[w_{h}\right]\right],\left[\left[w_{h}\right]\right]\right\rangle_{e} .
\end{aligned}
$$

Taking the real and imaginary parts yields

$$
\begin{aligned}
& \operatorname{Re} A_{h}\left(w_{h}, \chi_{h} ; w_{h},-\nabla_{h} w_{h}\right) \\
& =\left|w_{h}\right|_{1, h}^{2}-k^{2}\left\|w_{h}\right\|_{L^{2}(\Omega)}^{2}-2 \operatorname{Re} \sum_{e \in \mathcal{E}_{h}^{I}}\left\langle\left[\left[w_{h}\right]\right],\left\{\nabla_{h} w_{h}\right\}\right\rangle_{e}, \\
& \text { (3.8) } \quad \operatorname{Im} A_{h}\left(w_{h}, \boldsymbol{\chi}_{h} ; w_{h},-\nabla_{h} w_{h}\right) \\
& =\sum_{e \in \mathcal{E}_{h}^{I}}\left(\delta\left\|\left[\left[\nabla_{h} w_{h}\right]\right]\right\|_{L^{2}(e)}^{2}+\beta\left\|\left[\left[w_{h}\right]\right]\right\|_{L^{2}(e)}^{2}\right)+k\left\|w_{h}\right\|_{L^{2}(\Gamma)}^{2} .
\end{aligned}
$$

Step 2. Estimating $\left|w_{h}\right|_{1, h}^{2}$. Utilizing the fact that $\Delta u_{h}=0$ in $K \in \mathcal{T}_{h}$ for piecewise linear polynomial $u_{h}$, we have

$$
\begin{aligned}
\left|w_{h}\right|_{1, h}^{2}= & \sum_{K \in \mathcal{T}_{h}}\left(\nabla w_{h}, \nabla w_{h}\right)_{K}=\sum_{K \in \mathcal{T}_{h}} \int_{\partial K} \nabla_{h} w_{h} \cdot \mathbf{n}_{K} w_{h} d s \\
= & \sum_{e \in \mathcal{E}_{h}^{I}}\left(\left\langle\left\{\nabla_{h} w_{h}\right\},\left[\left[w_{h}\right]\right]\right\rangle_{e}+\left\langle\left[\left[\nabla_{h} w_{h}\right]\right],\left\{w_{h}\right\}\right\rangle_{e}\right)+\sum_{e \in \mathcal{E}_{h}^{B}}\left\langle\nabla_{h} w_{h} \cdot \mathbf{n}_{e}, w_{h}\right\rangle_{e} \\
\leq & \sum_{e \in \mathcal{E}_{h}^{I}}\left(\left\|\left\{\nabla_{h} w_{h}\right\}\right\|_{L^{2}(e)}\left\|\left[\left[w_{h}\right]\right]\right\|_{L^{2}(e)}+\left\|\left[\left[\nabla_{h} w_{h}\right]\right]\right\|_{L^{2}(e)}\left\|\left\{w_{h}\right\}\right\|_{L^{2}(e)}\right) \\
& +\sum_{e \in \mathcal{E}_{h}^{B}}\left\|\nabla_{h} w_{h} \cdot \mathbf{n}_{e}\right\|_{L^{2}(e)}\left\|w_{h}\right\|_{L^{2}(e)} .
\end{aligned}
$$

By the inverse inequality and the trace inequality [2], we have

$$
\begin{aligned}
\left|w_{h}\right|_{1, h}^{2} \leq & \sum_{e \in \mathcal{E}_{h}^{I}} h_{e}^{-\frac{1}{2}} \sum_{K=K_{e}, K_{e}^{\prime}}\left(\left\|\nabla w_{h}\right\|_{L^{2}(K)}\left\|\left[\left[w_{h}\right]\right]\right\|_{L^{2}(e)}\right. \\
& \left.+\left\|\left[\left[\nabla_{h} w_{h}\right]\right]\right\|_{L^{2}(e)}\left\|w_{h}\right\|_{L^{2}(K)}\right)+\sum_{e \in \mathcal{E}_{h}^{B}} h_{e}^{-\frac{1}{2}}\left\|\nabla_{h} w_{h} \cdot \mathbf{n}_{e}\right\|_{L^{2}(e)}\left\|w_{h}\right\|_{L^{2}\left(K_{e}\right)} \\
\leq & \frac{1}{2}\left|w_{h}\right|_{1, h}^{2}+\frac{k^{2}}{8}\left\|w_{h}\right\|_{\Omega}^{2}+C \sum_{e \in \mathcal{E}_{h}^{B}} \frac{1}{h_{e}}\left\|w_{h}\right\|_{L^{2}(e)}^{2} \\
& +C \sum_{e \in \mathcal{E}_{h}^{I}}\left(\frac{1}{h_{e}}\left\|\left[\left[w_{h}\right]\right]\right\|_{L^{2}(e)}^{2}+\frac{1}{k^{2} h_{e}}\left\|\left[\left[\nabla_{h} w_{h}\right]\right]\right\|_{L^{2}(e)}^{2}\right) .
\end{aligned}
$$


Therefore,

$$
\begin{aligned}
\left|w_{h}\right|_{1, h}^{2} \leq & \frac{k^{2}}{4}\left\|w_{h}\right\|_{L^{2}(\Omega)}^{2}+C \sum_{e \in \mathcal{E}_{h}^{B}} \frac{1}{h_{e}}\left\|w_{h}\right\|_{L^{2}(e)}^{2} \\
& +C \sum_{e \in \mathcal{E}_{h}^{I}}\left(\frac{1}{h_{e}}\left\|\left[\left[w_{h}\right]\right]\right\|_{L^{2}(e)}^{2}+\frac{1}{k^{2} h_{e}}\left\|\left[\left[\nabla_{h} w_{h}\right]\right]\right\|_{L^{2}(e)}^{2}\right) .
\end{aligned}
$$

Step 3. Finishing up. Following the same technique to derive the bound of $\left|w_{h}\right|_{1, h}^{2}$, we have

(3.10) $2 \operatorname{Re} \sum_{e \in \mathcal{E}_{h}^{I}}\left\langle\left[\left[w_{h}\right]\right],\left\{\nabla_{h} w_{h}\right\}\right\rangle_{e} \leq \frac{k^{2}}{4}\left\|w_{h}\right\|_{L^{2}(\Omega)}^{2}+C \sum_{e \in \mathcal{E}_{h}^{I}} \frac{1}{k^{2} h_{e}}\left\|\left[\left[\nabla_{h} w_{h}\right]\right]\right\|_{L^{2}(e)}^{2}$.

Combining (3.7), (3.9) and (3.10), we obtain

$$
\begin{aligned}
2\left|w_{h}\right|_{1, h}^{2}+\operatorname{Re} A_{h}\left(w_{h}, \boldsymbol{\chi}_{h} ; w_{h},-\nabla_{h} w_{h}\right) & \\
\leq & \frac{k^{2}}{2}\left\|w_{h}\right\|_{L^{2}(\Omega)}^{2}+C \sum_{e \in \mathcal{E}_{h}^{I}}\left(\frac{1}{h_{e}}\left\|\left[\left[w_{h}\right]\right]\right\|_{L^{2}(e)}^{2}+\frac{1}{k^{2} h_{e}}\left\|\left[\left[\nabla_{h} w_{h}\right]\right]\right\|_{L^{2}(e)}^{2}\right) \\
& +\left|w_{h}\right|_{1, h}^{2}-k^{2}\left\|w_{h}\right\|_{L^{2}(\Omega)}^{2}+\frac{k^{2}}{4}\left\|w_{h}\right\|_{L^{2}(\Omega)}^{2}+C \sum_{e \in \mathcal{E}_{h}^{B}} \frac{1}{h_{e}}\left\|w_{h}\right\|_{L^{2}(e)}^{2},
\end{aligned}
$$

which leads to

$$
\begin{aligned}
\left|w_{h}\right|_{1, h}^{2} & +k^{2}\left\|w_{h}\right\|_{L^{2}(\Omega)}^{2}+k\left\|w_{h}\right\|_{L^{2}(\Gamma)}^{2} \\
\leq & C\left(k+\max _{e \in \mathcal{E}_{h}^{B}} \frac{1}{h_{e}}\right)\left\|w_{h}\right\|_{L^{2}(\Gamma)}^{2}-4 \operatorname{Re} A_{h}\left(w_{h}, \chi_{h} ; w_{h},-\nabla_{h} w_{h}\right) \\
& +C \sum_{e \in \mathcal{E}_{h}^{I}}\left(\frac{1}{h_{e}}\left\|\left[\left[w_{h}\right]\right]\right\|_{L^{2}(e)}^{2}+\frac{1}{k^{2} h_{e}}\left\|\left[\left[\nabla_{h} w_{h}\right]\right]\right\|_{L^{2}(e)}^{2}\right)
\end{aligned}
$$

by adding $k\left\|w_{h}\right\|_{L^{2}(\Gamma)}^{2}$ on both sides.

Note that (3.8) provides upper bounds for the terms $\left\|\left[\left[\nabla_{h} w_{h}\right]\right]\right\|_{L^{2}(e)}^{2},\left\|\left[\left[w_{h}\right]\right]\right\|_{L^{2}(e)}^{2}$ and $k\left\|w_{h}\right\|_{L^{2}(\Gamma)}^{2}$ in terms of $\operatorname{Im} A_{h}\left(w_{h}, \chi_{h} ; w_{h},-\nabla_{h} w_{h}\right)$, using these bounds in (3.11) we get

$$
\begin{aligned}
\left|w_{h}\right|_{1, h}^{2} & +k^{2}\left\|w_{h}\right\|_{L^{2}(\Omega)}^{2}+k\left\|w_{h}\right\|_{L^{2}(\Gamma)}^{2} \\
& \lesssim-4 \operatorname{Re} A_{h}\left(w_{h}, \chi_{h} ; w_{h},-\nabla_{h} w_{h}\right)+\gamma_{1} \operatorname{Im} A_{h}\left(w_{h}, \chi_{h} ; w_{h},-\nabla_{h} w_{h}\right), \\
& \lesssim \gamma_{1}\left|A_{h}\left(w_{h}, \boldsymbol{\chi}_{h} ; w_{h},-\nabla_{h} w_{h}\right)\right|
\end{aligned}
$$

where $\gamma_{1}$ is defined by (3.5).

By the definition of $\|\cdot\|_{D G}$ in (3.6), we have

$$
\frac{\left|A_{h}\left(w_{h}, \chi_{h} ; w_{h},-\nabla_{h} w_{h}\right)\right|}{\left\|w_{h}\right\|_{D G}} \geq \frac{c_{1}}{\gamma_{1}}\left\|w_{h}\right\|_{D G} .
$$

Hence, (3.4) holds. The proof is complete.

Remark 3.1. (a) We note that $\gamma_{1}$ depends on both $h$ and $k$ and

$$
\gamma_{1}=1+\max _{e \in \mathcal{E}_{h}^{B}} \frac{1}{k h_{e}}+\max _{e \in \mathcal{E}_{h}^{I}}\left(1+\frac{1}{k^{2} h_{e}^{2}}\right),
$$


if choosing $\beta=h_{e}^{-1}$ and $\delta=h_{e}$.

(b) Unlike in [10, 11, (3.4) holds without assuming that $\Omega$ is a star-shaped domain.

3.1.2. Stability estimates. The goal of this subsection is to establish the absolute stability for the LDG method \#1 using the inf-sup condition proved in the previous subsection.

Theorem 3.1. Let $\left(u_{h}, \boldsymbol{\sigma}_{h}\right) \in V_{h} \times \boldsymbol{\Sigma}_{h}$ solve (3.1). Define

$$
M(f, g):=\|f\|_{\Omega}+\sqrt{k}\|g\|_{L^{2}(\Gamma)} .
$$

Then for all $k, h, \beta, \delta>0$ the following stability estimates hold:

$$
\begin{aligned}
\left\|u_{h}\right\|_{D G} & \lesssim \gamma_{1} k^{-1} M(f, g), \\
\left\|\boldsymbol{\sigma}_{h}\right\|_{L^{2}(\Omega)} & \lesssim \gamma_{1} k^{-1}\left(1+\left(\delta+k^{-1}\right)\left(\max _{K \in \mathcal{T}_{h}} h_{K}^{-1}\right)\right) M(f, g) .
\end{aligned}
$$

Proof. By Schwarz inequality we have

$$
\begin{aligned}
\left|F\left(v_{h}, \chi_{h}\right)\right| & \leq\|f\|_{L^{2}(\Omega)}\left\|v_{h}\right\|_{L^{2}(\Omega)}+\|g\|_{L^{2}(\Gamma)}\left\|v_{h}\right\|_{L^{2}(\Gamma)} \\
& \leq C k^{-1} M(f, g)\left(k^{2}\left\|v_{h}\right\|_{L^{2}(\Omega)}^{2}+k\left\|v_{h}\right\|_{L^{2}(\Gamma)}^{2}\right)^{\frac{1}{2}} . \\
& \leq C k^{-1} M(f, g)\left\|v_{h}\right\|_{D G} .
\end{aligned}
$$

Let $\left(w_{h}, \boldsymbol{\chi}_{h}\right)=\left(u_{h}, \boldsymbol{\sigma}_{h}\right)$ in (3.4). By equation (3.1) and (3.16) we get

$$
\begin{aligned}
\frac{c_{1}}{\gamma_{1}}\left\|u_{h}\right\|_{D G} & \leq \sup _{\left(v_{h}, \boldsymbol{\tau}_{h}\right) \in V_{h} \times \boldsymbol{\Sigma}_{h}} \frac{\left|A_{h}\left(u_{h}, \boldsymbol{\sigma}_{h} ; v_{h}, \boldsymbol{\tau}_{h}\right)\right|}{\left\|v_{h}\right\|_{D G}} \\
& =\sup _{\left(v_{h}, \boldsymbol{\tau}_{h}\right) \in V_{h} \times \boldsymbol{\Sigma}_{h}} \frac{\left|F\left(v_{h}, \boldsymbol{\tau}_{h}\right)\right|}{\left\|v_{h}\right\|_{D G}} \\
& \leq C k^{-1} M(f, g) .
\end{aligned}
$$

Hence (3.14) holds.

To show (3.15), setting $\left(v_{h}, \boldsymbol{\tau}_{h}\right)=\left(0, \boldsymbol{\sigma}_{h}\right)$ in (3.1) and using the trace and Schwarz inequalities yields

$$
\begin{gathered}
\left\|\boldsymbol{\sigma}_{h}\right\|_{L^{2}(\Omega)}^{2}=\left(\nabla_{h} u_{h}, \boldsymbol{\sigma}_{h}\right)_{\Omega}+\sum_{e \in \mathcal{E}_{h}^{I}}\left(\mathbf{i} \delta\left\langle\left[\left[\nabla_{h} u_{h}\right]\right],\left[\left[\boldsymbol{\sigma}_{h}\right]\right]\right\rangle_{e}-\left\langle\left[\left[u_{h}\right]\right],\left\{\boldsymbol{\sigma}_{h}\right\}\right\rangle_{e}\right) \\
\leq\left\|\nabla_{h} u_{h}\right\|_{L^{2}(\Omega)}^{2}+\frac{1}{4}\left\|\boldsymbol{\sigma}_{h}\right\|_{L^{2}(\Omega)}^{2}+C \sum_{e \in \mathcal{E}_{h}^{I}} \sum_{K=K_{e}, K_{e}^{\prime}} h_{K}^{-1}\left(\delta\left\|\nabla u_{h}\right\|_{L^{2}(K)}\right. \\
\left.+\left\|u_{h}\right\|_{L^{2}(K)}\right)\left\|\boldsymbol{\sigma}_{h}\right\|_{L^{2}(K)} \\
\leq\left\|\nabla_{h} u_{h}\right\|_{L^{2}(\Omega)}^{2}+\frac{1}{2}\left\|\boldsymbol{\sigma}_{h}\right\|_{L^{2}(\Omega)}^{2} \quad \\
+C \sum_{e \in \mathcal{E}_{h}^{I}} \sum_{K=K_{e}, K_{e}^{\prime}} h_{K}^{-2}\left(\delta^{2}\left\|\nabla u_{h}\right\|_{L^{2}(K)}^{2}+\left\|u_{h}\right\|_{L^{2}(K)}^{2}\right)
\end{gathered}
$$

Thus,

$$
\left\|\boldsymbol{\sigma}_{h}\right\|_{L^{2}(\Omega)}^{2} \lesssim\left(1+\delta^{2}\left(\max _{K \in \mathcal{T}_{h}} h_{K}^{-2}\right)\right)\left|u_{h}\right|_{1, h}^{2}+\left(\max _{K \in \mathcal{T}_{h}} h_{K}^{-2}\right)\left\|u_{h}\right\|_{L^{2}(\Omega)}^{2} .
$$

The desired estimate (3.15) follows from combining the above inequality with (3.14). The proof is complete. 
An immediate consequence of the stability estimates is the following unique solvability theorem.

Theorem 3.2. There exists a unique solution to the $L D G$ method (3.1) for all $k, h, \delta, \beta>0$.

Proof. Since problem (3.1) is equivalent to a linear system, it suffices to show the uniqueness. But the uniqueness follows immediately from the stability estimates as the zero sources imply that any solution must be a trivial solution.

3.2. Absolute stability of LDG method \#2. In this subsection, we consider the LDG method \#2. By adding (2.7) and (2.8) with the given numerical fluxes, we then recast the LDG method \#2 as the following nonconforming mixed method: Find $\left(u_{h}, \boldsymbol{\sigma}_{h}\right) \in V_{h} \times \boldsymbol{\Sigma}_{h}$ such that

$$
B_{h}\left(u_{h}, \boldsymbol{\sigma}_{h} ; v_{h}, \boldsymbol{\tau}_{h}\right)=F\left(v_{h}, \boldsymbol{\tau}_{h}\right) \quad \forall\left(v_{h}, \boldsymbol{\tau}_{h}\right) \in V_{h} \times \boldsymbol{\Sigma}_{h},
$$

where $F$ is defined in (3.3) and

$$
\begin{aligned}
& B_{h}\left(w_{h}, \boldsymbol{\chi}_{h} ; v_{h}, \boldsymbol{\tau}_{h}\right)=\left(\boldsymbol{\chi}_{h}, \nabla_{h} v_{h}\right)_{\Omega}-k^{2}\left(w_{h}, v_{h}\right)_{\Omega}+\mathbf{i} k\left\langle w_{h}, v_{h}\right\rangle_{\Gamma} \\
&-\sum_{e \in \mathcal{E}_{h}^{I}}\left\langle\left\{\boldsymbol{\chi}_{h}\right\}-\mathbf{i} \beta\left[\left[w_{h}\right]\right],\left[\left[v_{h}\right]\right]\right\rangle_{e} \\
&-\sum_{e \in \mathcal{E}_{h}^{I}}\left(\mathbf{i} \delta\left\langle\left[\left[\boldsymbol{\chi}_{h}\right]\right],\left[\left[\boldsymbol{\tau}_{h}\right]\right]\right\rangle_{e}-\left\langle\left[\left[w_{h}\right]\right],\left\{\boldsymbol{\tau}_{h}\right\}\right\rangle_{e}\right) \\
&+\left(\boldsymbol{\chi}_{h}, \boldsymbol{\tau}_{h}\right)_{\Omega}-\left(\nabla_{h} w_{h}, \boldsymbol{\tau}_{h}\right)_{\Omega} .
\end{aligned}
$$

3.2.1. Coercivity property of $B_{h}$. The goal of this subsection is to show that the sesquilinear form $B_{h}$ defined in (3.18) for the LDG method \#2 is coercive. To this end, we introduce the following space notation:

$$
\left.\mathcal{S}_{h}:=\left\{\left(w_{h}, \chi_{h}\right) \in V_{h} \times \boldsymbol{\Sigma}_{h} ;\left(w_{h}, \chi_{h}\right) \text { satisfies } 3.20\right)\right\},
$$

where

$$
\begin{aligned}
& \left(\boldsymbol{\chi}_{h}, \boldsymbol{\tau}_{h}\right)_{\Omega}-\left(\nabla_{h} w_{h}, \boldsymbol{\tau}_{h}\right)_{\Omega} \\
& \quad-\sum_{e \in \mathcal{E}_{h}^{I}}\left(\mathbf{i} \delta\left\langle\left[\left[\boldsymbol{\chi}_{h}\right]\right],\left[\left[\boldsymbol{\tau}_{h}\right]\right]\right\rangle_{e}-\left\langle\left[\left[w_{h}\right]\right],\left\{\boldsymbol{\tau}_{h}\right\}\right\rangle_{e}\right)=0 \quad \forall \boldsymbol{\tau}_{h} \in \boldsymbol{\Sigma}_{h} .
\end{aligned}
$$

The space $\mathcal{S}_{h}$ is well defined and is not an empty set, as $(c, 0) \in \mathcal{S}_{h}$ for any constant $c$. Next, we note that it is easy to check that $\left(w_{h}, \chi_{h}\right) \in \mathcal{S}_{h}$ implies that it satisfies (2.7) with $\hat{u}_{K}$ being defined by the LDG method \#2.

Lemma 3.1. For any $\left(w_{h}, \chi_{h}\right) \in \mathcal{S}_{h}$, the following estimates hold:

$$
\begin{aligned}
&\left|w_{h}\right|_{1, h} \leq \sqrt{\frac{17}{16}}\left\|\chi_{h}\right\|_{L^{2}(\Omega)} \\
&+C\left(\sum_{e \in \mathcal{E}_{h}^{I}}\left(\frac{1}{h_{e}}\left\|\left[\left[w_{h}\right]\right]\right\|_{L^{2}(e)}^{2}+\frac{\delta^{2}}{h_{e}}\left\|\left[\left[\chi_{h}\right]\right]\right\|_{L^{2}(e)}^{2}\right)\right)^{\frac{1}{2}} \\
&\left\|\chi_{h}-\nabla_{h} w_{h}\right\|_{L^{2}(\Omega)} \leq C\left(\sum_{e \in \mathcal{E}_{h}^{I}}\left(\frac{1}{h_{e}}\left\|\left[\left[w_{h}\right]\right]\right\|_{L^{2}(e)}^{2}+\frac{\delta^{2}}{h_{e}}\left\|\left[\left[\chi_{h}\right]\right]\right\|_{L^{2}(e)}^{2}\right)\right)^{\frac{1}{2}} .
\end{aligned}
$$


Proof. Note that $\left(w_{h}, \chi_{h}\right)$ satisfies (3.20); thus, setting $\tau_{h}=\nabla_{h} w_{h}$ in (3.20), we get

$$
\begin{aligned}
\left|w_{h}\right|_{1, h}^{2}= & \operatorname{Re}\left(\chi_{h}, \nabla_{h} w_{h}\right)_{\Omega} \\
& -\operatorname{Re} \sum_{e \in \mathcal{E}_{h}^{I}}\left(\mathbf{i} \delta\left\langle\left[\left[\chi_{h}\right]\right],\left[\left[\nabla_{h} w_{h}\right]\right]\right\rangle_{e}-\left\langle\left[\left[w_{h}\right]\right],\left\{\nabla_{h} w_{h}\right\}\right\rangle_{e}\right) \\
\leq & \frac{1}{2}\left|w_{h}\right|_{1, h}^{2}+\frac{1}{2}\left\|\chi_{h}\right\|_{L^{2}(\Omega)}^{2} \\
& +\sum_{e \in \mathcal{E}_{h}^{I}} \delta h_{e}^{-\frac{1}{2}} \sum_{K=K_{e}, K_{e}^{\prime}}\left\|\left[\left[\chi_{h}\right]\right]\right\|_{L^{2}(e)}\left\|\nabla w_{h}\right\|_{L^{2}(K)} \\
& +\sum_{e \in \mathcal{E}_{h}^{I}} h_{e}^{-\frac{1}{2}} \sum_{K=K_{e}, K_{e}^{\prime}}\left\|\left[\left[w_{h}\right]\right]\right\|_{L^{2}(e)}\left\|\nabla w_{h}\right\|_{L^{2}(K)} \\
\leq \frac{1}{2}\left|w_{h}\right|_{1, h}^{2}+\frac{1}{2}\left\|\chi_{h}\right\|_{L^{2}(\Omega)}^{2}+\frac{1}{34}\left|w_{h}\right|_{1, h}^{2} & +C \sum_{e \in \mathcal{E}_{h}^{I}}\left(\frac{1}{h_{e}}\left\|\left[\left[w_{h}\right]\right]\right\|_{L^{2}(e)}^{2}+\frac{\delta^{2}}{h_{e}}\left\|\left[\left[\chi_{h}\right]\right]\right\|_{L^{2}(e)}^{2}\right) .
\end{aligned}
$$

Therefore,

$$
\left|w_{h}\right|_{1, h}^{2} \leq \frac{17}{16}\left\|\chi_{h}\right\|_{L^{2}(\Omega)}^{2}+C \sum_{e \in \mathcal{E}_{h}^{I}}\left(\frac{1}{h_{e}}\left\|\left[\left[w_{h}\right]\right]\right\|_{L^{2}(e)}^{2}+\frac{\delta^{2}}{h_{e}}\left\|\left[\left[\chi_{h}\right]\right]\right\|_{L^{2}(e)}^{2}\right),
$$

which gives (3.21).

The estimate (3.22) follows from the same derivation by setting $\boldsymbol{\tau}_{h}=\chi_{h}-\nabla_{h} w_{h}$ in (3.20). The proof is complete.

We now are ready to state a coercivity property for the sesquilinear form $B_{h}$.

Proposition 3.2. There exists the constant $c_{2}>0$ such that for any $\left(w_{h}, \chi_{h}\right) \in \mathcal{S}_{h}$ it holds that

$$
\left|B_{h}\left(w_{h}, \chi_{h} ; w_{h}, \chi_{h}\right)\right| \geq \frac{c_{2}}{\gamma_{2}}\left\|\left(w_{h}, \chi_{h}\right)\right\|_{D G}^{2},
$$

where

$$
\begin{gathered}
\gamma_{2}:=1+\max _{e \in \mathcal{E}_{h}^{B}} \frac{1}{k h_{e}}+\max _{e \in \mathcal{E}_{h}^{I}}\left(\frac{1}{\beta k^{2} h_{e}^{3}}+\frac{1}{\beta h_{e}}+\frac{\delta}{h_{e}}+\frac{\delta}{k^{2} h_{e}^{3}}+\frac{1}{\delta k^{2} h_{e}}\right), \\
\left\|\left(w_{h}, \chi_{h}\right)\right\|_{D G}:=\left(k^{2}\left\|w_{h}\right\|_{L^{2}(\Omega)}^{2}+k\left\|w_{h}\right\|_{L^{2}(\Gamma)}^{2}+\left\|\chi_{h}\right\|_{L^{2}(\Omega)}^{2}\right)^{\frac{1}{2}} .
\end{gathered}
$$

Proof. Since the proof follows the same lines as that of Proposition 3.1, we shall only highlight the main steps and point out the differences. 
Step 1. Taking the test function. We first choose the test function $\left(v_{h}, \boldsymbol{\tau}_{h}\right)=$ $\left(w_{h}, \chi_{h}\right)$ to get

$$
\begin{aligned}
B_{h}\left(w_{h}, \boldsymbol{\chi}_{h} ; v_{h}, \boldsymbol{\tau}_{h}\right)= & B_{h}\left(w_{h}, \chi_{h} ; w_{h}, \chi_{h}\right) \\
= & \left(\boldsymbol{\chi}_{h}, \chi_{h}\right)_{\Omega}-k^{2}\left(w_{h}, w_{h}\right)_{\Omega}+\mathbf{i} k\left\langle w_{h}, w_{h}\right\rangle_{\Gamma} \\
& -\sum_{e \in \mathcal{E}_{h}^{I}}\left\langle\left\{\chi_{h}\right\}-\mathbf{i} \beta\left[\left[w_{h}\right]\right],\left[\left[w_{h}\right]\right]\right\rangle_{e} \\
& -\sum_{e \in \mathcal{E}_{h}^{I}}\left(\mathbf{i} \delta\left\langle\left[\left[\chi_{h}\right]\right],\left[\left[\boldsymbol{\chi}_{h}\right]\right]\right\rangle_{e}-\left\langle\left[\left[w_{h}\right]\right],\left\{\chi_{h}\right\}\right\rangle_{e}\right) \\
& +\left(\chi_{h}, \nabla_{h} w_{h}\right)_{\Omega}-\left(\nabla_{h} w_{h}, \boldsymbol{\chi}_{h}\right)_{\Omega} .
\end{aligned}
$$

Taking the real and imaginary parts yields

$$
\begin{aligned}
\operatorname{Re} B_{h}\left(w_{h}, \boldsymbol{\chi}_{h} ; w_{h}, \boldsymbol{\chi}_{h}\right)= & \left\|\boldsymbol{\chi}_{h}\right\|_{L^{2}(\Omega)}^{2}-k^{2}\left\|w_{h}\right\|_{L^{2}(\Omega)}^{2} \\
\operatorname{Im} B_{h}\left(w_{h}, \boldsymbol{\chi}_{h} ; w_{h}, \boldsymbol{\chi}_{h}\right)= & k\left\|w_{h}\right\|_{L^{2}(\Gamma)}^{2} \\
& +\sum_{e \in \mathcal{E}_{h}^{I}}\left(-\delta\left\|\left[\left[\boldsymbol{\chi}_{h}\right]\right]\right\|_{L^{2}(e)}^{2}+\beta\left\|\left[\left[w_{h}\right]\right]\right\|_{L^{2}(e)}^{2}\right) \\
& +2 \operatorname{Im}\left(\boldsymbol{\chi}_{h}, \nabla_{h} w_{h}\right)_{\Omega}+\sum_{e \in \mathcal{E}_{h}^{I}} 2 \operatorname{Im}\left\langle\left[\left[w_{h}\right]\right],\left\{\boldsymbol{\chi}_{h}\right\}\right\rangle_{e} .
\end{aligned}
$$

Note that $\left(w_{h}, \chi_{h}\right)$ satisfies (3.20); thus, setting $\tau_{h}=\chi_{h}$ in (3.20), we get

$$
\left\|\chi_{h}\right\|_{L^{2}(\Omega)}^{2}-\left(\nabla_{h} w_{h}, \chi_{h}\right)_{\Omega}=\sum_{e \in \mathcal{E}_{h}^{I}}\left(\mathbf{i} \delta\left\|\left[\left[\chi_{h}\right]\right]\right\|_{L^{2}(e)}^{2}-\left\langle\left[\left[w_{h}\right]\right],\left\{\chi_{h}\right\}\right\rangle_{e}\right) .
$$

Taking the imaginary part yields

$$
\operatorname{Im}\left(\boldsymbol{\chi}_{h}, \nabla_{h} w_{h}\right)_{\Omega}+\sum_{e \in \mathcal{E}_{h}^{I}} \operatorname{Im}\left\langle\left[\left[w_{h}\right]\right],\left\{\boldsymbol{\chi}_{h}\right\}\right\rangle_{e}=\sum_{e \in \mathcal{E}_{h}^{I}} \delta\left\|\left[\left[\boldsymbol{\chi}_{h}\right]\right]\right\|_{L^{2}(e)}^{2} .
$$

Hence, (3.27) becomes

$$
\begin{aligned}
\operatorname{Im} B_{h}\left(w_{h}, \boldsymbol{\chi}_{h} ; w_{h}, \boldsymbol{\chi}_{h}\right)= & k\left\|w_{h}\right\|_{L^{2}(\Gamma)}^{2} \\
& +\sum_{e \in \mathcal{E}_{h}^{I}}\left(\delta\left\|\left[\left[\boldsymbol{\chi}_{h}\right]\right]\right\|_{L^{2}(e)}^{2}+\beta\left\|\left[\left[w_{h}\right]\right]\right\|_{L^{2}(e)}^{2}\right) .
\end{aligned}
$$

Step 2. Estimating $\left\|\chi_{h}\right\|_{L^{2}(\Omega)}^{2}$. By (3.9) and (3.22) we have

$$
\begin{gathered}
\left\|\chi_{h}\right\|_{L^{2}(\Omega)}^{2} \leq 3\left\|\chi_{h}-\nabla_{h} w_{h}\right\|_{L^{2}(\Omega)}^{2}+\frac{3}{2}\left|w_{h}\right|_{1, h}^{2} \\
\leq \frac{3 k^{2}}{8}\left\|w_{h}\right\|_{L^{2}(\Omega)}^{2}+C \sum_{e \in \mathcal{E}_{h}^{B}} \frac{1}{h_{e}}\left\|w_{h}\right\|_{L^{2}(e)}^{2}+C \sum_{e \in \mathcal{E}_{h}^{I}}\left(\frac{1}{h_{e}}\left\|\left[\left[w_{h}\right]\right]\right\|_{L^{2}(e)}^{2}\right. \\
\left.\quad+\frac{1}{k^{2} h_{e}}\left\|\left[\left[\nabla_{h} w_{h}\right]\right]\right\|_{L^{2}(e)}^{2}+\frac{\delta^{2}}{h_{e}}\left\|\left[\left[\chi_{h}\right]\right]\right\|_{L^{2}(e)}^{2}\right)
\end{gathered}
$$


Using the estimate

$$
\begin{aligned}
\sum_{e \in \mathcal{E}_{h}^{I}}\left\|\left[\left[\nabla_{h} w_{h}\right]\right]\right\|_{L^{2}(e)}^{2} & \leq 2 \sum_{e \in \mathcal{E}_{h}^{I}}\left\|\left[\left[\chi_{h}\right]\right]\right\|_{L^{2}(e)}^{2}+2 \sum_{e \in \mathcal{E}_{h}^{I}}\left\|\left[\left[\nabla_{h} w_{h}-\chi_{h}\right]\right]\right\|_{L^{2}(e)}^{2} \\
& \lesssim \sum_{e \in \mathcal{E}_{h}^{I}}\left\|\left[\left[\chi_{h}\right]\right]\right\|_{L^{2}(e)}^{2}+\sum_{e \in \mathcal{E}_{h}^{I}} h_{e}^{-1} \sum_{K=K_{e}, K_{e}^{\prime}}\left\|\nabla w_{h}-\chi_{h}\right\|_{L^{2}(K)}^{2} \\
& \lesssim \sum_{e \in \mathcal{E}_{h}^{I}}\left\|\left[\left[\chi_{h}\right]\right]\right\|_{L^{2}(e)}^{2}+\max _{e \in \mathcal{E}_{h}} h_{e}^{-1}\left\|\nabla_{h} w_{h}-\chi_{h}\right\|_{L^{2}(\Omega)}^{2} \\
& \lesssim \sum_{e \in \mathcal{E}_{h}^{I}} \frac{\delta^{2}+h_{e}^{2}}{h_{e}^{2}}\left\|\left[\left[\chi_{h}\right]\right]\right\|_{L^{2}(e)}^{2}+\sum_{e \in \mathcal{E}_{h}^{I}} \frac{1}{h_{e}^{2}}\left\|\left[\left[w_{h}\right]\right]\right\|_{L^{2}(e)}^{2},
\end{aligned}
$$

we get

$$
\begin{aligned}
& \left\|\chi_{h}\right\|_{L^{2}(\Omega)}^{2} \leq \frac{3 k^{2}}{8}\left\|w_{h}\right\|_{L^{2}(\Omega)}^{2}+C \sum_{e \in \mathcal{E}_{h}^{B}} \frac{1}{h_{e}}\left\|w_{h}\right\|_{L^{2}(e)}^{2} \\
& \quad+C \sum_{e \in \mathcal{E}_{h}^{I}}\left(\frac{1+k^{2} h_{e}^{2}}{k^{2} h_{e}^{3}}\left\|\left[\left[w_{h}\right]\right]\right\|_{L^{2}(e)}^{2}+\frac{\delta^{2}\left(1+k^{2} h_{e}^{2}\right)+h_{e}^{2}}{k^{2} h_{e}^{3}}\left\|\left[\left[\chi_{h}\right]\right]\right\|_{L^{2}(e)}^{2}\right) .
\end{aligned}
$$

Step 3. Finishing up. It follows from (3.26) and (3.30) that

$$
\begin{aligned}
& \operatorname{Re} B_{h}\left(w_{h}, \chi_{h} ; w_{h}, \chi_{h}\right)+2\left\|\chi_{h}\right\|_{L^{2}(\Omega)}^{2} \\
& \leq\left\|\chi_{h}\right\|_{L^{2}(\Omega)}^{2}-k^{2}\left\|w_{h}\right\|_{L^{2}(\Omega)}^{2}+\frac{3 k^{2}}{4}\left\|w_{h}\right\|_{L^{2}(\Omega)}^{2}+C \sum_{e \in \mathcal{E}_{h}^{B}} \frac{1}{h_{e}}\left\|w_{h}\right\|_{L^{2}(e)}^{2} \\
& \quad+C \sum_{e \in \mathcal{E}_{h}^{I}}\left(\frac{1+k^{2} h_{e}^{2}}{k^{2} h_{e}^{3}}\left\|\left[\left[w_{h}\right]\right]\right\|_{L^{2}(e)}^{2}+\frac{\delta^{2}\left(1+k^{2} h_{e}^{2}\right)+h_{e}^{2}}{k^{2} h_{e}^{3}}\left\|\left[\left[\chi_{h}\right]\right]\right\|_{L^{2}(e)}^{2}\right),
\end{aligned}
$$

which leads to

$$
\begin{aligned}
& \left\|\chi_{h}\right\|_{L^{2}(\Omega)}^{2}+k^{2}\left\|w_{h}\right\|_{L^{2}(\Omega)}^{2}+k\left\|w_{h}\right\|_{L^{2}(\Gamma)}^{2} \\
& \quad \leq C\left(k+\max _{e \in \mathcal{E}_{h}^{B}} \frac{1}{h_{e}}\right)\left\|w_{h}\right\|_{L^{2}(\Gamma)}^{2}-4 \operatorname{Re} B_{h}\left(w_{h}, \chi_{h} ; w_{h}, \chi_{h}\right) \\
& \left.\quad+C \sum_{e \in \mathcal{E}_{h}^{I}}\left(\frac{1+k^{2} h_{e}^{2}}{k^{2} h_{e}^{3}}\left\|\left[\left[w_{h}\right]\right]\right\|_{L^{2}(e)}^{2}+\frac{\delta^{2}\left(1+k^{2} h_{e}^{2}\right)+h_{e}^{2}}{k^{2} h_{e}^{3}} \|\left[\chi_{h}\right]\right] \|_{L^{2}(e)}^{2}\right)
\end{aligned}
$$

by adding $k\left\|w_{h}\right\|_{L^{2}(\Gamma)}^{2}$ on both sides.

Note that (3.28) provides upper bounds for the terms $\left\|\left[\left[\chi_{h}\right]\right]\right\|_{L^{2}(e)}^{2},\left\|\left[\left[w_{h}\right]\right]\right\|_{L^{2}(e)}^{2}$ and $k\left\|w_{h}\right\|_{L^{2}(\Gamma)}^{2}$ in terms of $\operatorname{Im} B_{h}\left(w_{h}, \boldsymbol{\chi}_{h} ; w_{h}, \boldsymbol{\chi}_{h}\right)$; thus, using these bounds in (3.31) we get

$$
\begin{aligned}
\|\chi\|_{L^{2}(\Omega)}^{2}+k^{2}\left\|w_{h}\right\|_{L^{2}(\Omega)}^{2}+k\left\|w_{h}\right\|_{L^{2}(\Gamma)}^{2} & \\
& \lesssim-4 \operatorname{Re} B_{h}\left(w_{h}, \boldsymbol{\chi}_{h} ; w_{h}, \boldsymbol{\chi}_{h}\right)+\gamma_{2} \operatorname{Im} B_{h}\left(w_{h}, \chi_{h} ; w_{h}, \boldsymbol{\chi}_{h}\right), \\
& \lesssim \gamma_{2}\left|B_{h}\left(w_{h}, \boldsymbol{\chi}_{h} ; w_{h}, \boldsymbol{\chi}_{h}\right)\right|
\end{aligned}
$$

where $\gamma_{2}$ is defined by (3.24). 
By the definition of $\|\cdot\|_{D G}$ in 3.25), we have

$$
\left|B_{h}\left(w_{h}, \chi_{h} ; w_{h}, \chi_{h}\right)\right| \geq \frac{c_{2}}{\gamma_{2}}\left\|\left(w_{h}, \chi_{h}\right)\right\|_{D G}^{2}
$$

Hence, 3.23 holds. The proof is complete.

Remark 3.2. We note that $\gamma_{2}$ depends on both $h$ and $k$ and

$$
\gamma_{2}=1+\max _{e \in \mathcal{E}_{h}^{B}} \frac{1}{k h_{e}}+\max _{e \in \mathcal{E}_{h}^{I}}\left(1+\frac{1}{k^{2} h_{e}^{2}}\right)
$$

if setting $\beta=h_{e}^{-1}$ and $\delta=h_{e}$.

3.2.2. Stability estimates. The coercivity property proved in the last subsection immediately infers the following stability and well-posedness theorems for the LDG method \#2.

Theorem 3.3. Let $\left(u_{h}, \boldsymbol{\sigma}_{h}\right) \in V_{h} \times \boldsymbol{\Sigma}_{h}$ solve (3.17). Then for all $k, h, \beta, \delta>0$ it holds that

$$
\left\|\left(u_{h}, \boldsymbol{\sigma}_{h}\right)\right\|_{D G} \lesssim \gamma_{2} k^{-1} M(f, g) .
$$

Proof. Note that if any solution $\left(u_{h}, \boldsymbol{\sigma}_{h}\right)$ of (3.17) belongs to the set $\mathcal{S}_{h}$, the desired estimate (3.33) follows readily from (3.23) with $\left(w_{h}, \chi_{h}\right)=\left(u_{h}, \boldsymbol{\sigma}_{h}\right)$, (3.17) and (3.16). The proof is complete.

Theorem 3.4. The LDG method (3.17) has a unique solution for all $k, h, \delta, \beta>0$.

\section{ERror estimates}

The goal of this section is to derive error estimates for the LDG methods \#1 and \#2. Following the idea of [10, this will be done in two steps. First, we introduce an elliptic projection of the solution $(u, \sigma)$ using a corresponding coercive sesquilinear form of $A_{h}$ (resp. $B_{h}$ ) and derive error bounds for the projection. We note that the error analysis for the elliptic projections has an independent interest in itself (cf. [3). Second, we bound the error between the projection and the LDG solution using the stability estimates obtained in Section 3 . Since the error analysis for the two LDG methods are similar, we shall give more details of the error analysis for the LDG method \#1 but shall be brief for the LDG method \#2. Throughout this section, for $j=1,2$, we let

$$
H^{j}\left(\mathcal{T}_{h}\right)=\prod_{K \in \mathcal{T}_{h}} H^{j}(K), \quad h=\max _{K \in \mathcal{T}_{h}} h_{K} \approx \max _{e \in \mathcal{E}_{h}} h_{e}, \quad \beta=\beta_{0} h^{-1}, \quad \delta=\delta_{0} h
$$

for some positive constants $\beta_{0}$ and $\delta_{0}$, and assume $\mathcal{T}_{h}$ is quasi-uniform. For convenience, $\Omega$ is assumed to be polygonal. 


\subsection{Error estimates for the LDG method \#1.}

4.1.1. Elliptic projection and its error estimates. For any $(w, \chi) \in H^{2}\left(\mathcal{T}_{h}\right) \times$ $H^{1}\left(\mathcal{T}_{h}\right)^{d}$, we define the elliptic projection $\left(\tilde{w}_{h}, \tilde{\chi}_{h}\right) \in V_{h} \times \boldsymbol{\Sigma}_{h}$ of $(w, \chi)$ by

$$
a_{h}\left(\tilde{w}_{h}, \tilde{\chi}_{h} ; v_{h}, \boldsymbol{\tau}_{h}\right)=a_{h}\left(w, \boldsymbol{\chi} ; v_{h}, \boldsymbol{\tau}_{h}\right) \quad \forall\left(v_{h}, \boldsymbol{\tau}_{h}\right) \in V_{h} \times \boldsymbol{\Sigma}_{h}
$$

where

$$
\begin{aligned}
a_{h}\left(w_{h}, \chi_{h} ; v_{h}, \boldsymbol{\tau}_{h}\right):= & A_{h}\left(w_{h}, \boldsymbol{\chi}_{h} ; v_{h}, \boldsymbol{\tau}_{h}\right)+k^{2}\left(w_{h}, v_{h}\right)_{\Omega} \\
=\left(\boldsymbol{\chi}_{h}, \nabla_{h} v_{h}\right)_{\Omega}+\mathbf{i} k\left\langle w_{h}, v_{h}\right\rangle_{\Gamma} & -\sum_{e \in \mathcal{E}_{h}^{I}}\left\langle\left\{\nabla_{h} w_{h}\right\}-\mathbf{i} \beta_{0} h^{-1}\left[\left[w_{h}\right]\right],\left[\left[v_{h}\right]\right]\right\rangle_{e} \\
& -\sum_{e \in \mathcal{E}_{h}^{I}}\left(\mathbf{i} \delta\left\langle\left[\left[\nabla_{h} w_{h}\right]\right],\left[\left[\boldsymbol{\tau}_{h}\right]\right]\right\rangle_{e}-\left\langle\left[\left[w_{h}\right]\right],\left\{\boldsymbol{\tau}_{h}\right\}\right\rangle_{e}\right) \\
& +\left(\boldsymbol{\chi}_{h}, \boldsymbol{\tau}_{h}\right)_{\Omega}-\left(\nabla_{h} w_{h}, \boldsymbol{\tau}_{h}\right)_{\Omega} .
\end{aligned}
$$

To derive error bounds for the above elliptic projection, we first notice that

$$
\begin{aligned}
a_{h}\left(w_{h}, \chi_{h} ; v_{h},-\nabla_{h} v_{h}\right)= & \left(\nabla_{h} w_{h}, \nabla_{h} v_{h}\right)_{\Omega}+\mathbf{i} k\left\langle w_{h}, v_{h}\right\rangle_{\Gamma} \\
& \quad-\sum_{e \in \mathcal{E}_{h}^{I}}\left\langle\left\{\nabla_{h} w_{h}\right\}-\mathbf{i} \beta_{0} h^{-1}\left[\left[w_{h}\right]\right],\left[\left[v_{h}\right]\right]\right\rangle_{e} \\
& +\sum_{e \in \mathcal{E}_{h}^{I}}\left(\mathbf{i} \delta\left\langle\left[\left[\nabla_{h} w_{h}\right]\right],\left[\left[\nabla_{h} v_{h}\right]\right]\right\rangle_{e}-\left\langle\left[\left[w_{h}\right]\right],\left\{\nabla_{h} v_{h}\right\}\right\rangle_{e}\right) \\
=: & \mathcal{A}_{h}\left(w_{h}, v_{h}\right) .
\end{aligned}
$$

As a result, $\tilde{w}_{h} \in V_{h}$ satisfies

$$
\mathcal{A}_{h}\left(\tilde{w}_{h}, v_{h}\right)=\mathcal{A}_{h}\left(w, v_{h}\right) \quad \forall v_{h} \in V_{h} .
$$

Moreover, since

$$
\begin{aligned}
a_{h}\left(w_{h}, \chi_{h} ; 0, \boldsymbol{\tau}_{h}\right)=( & \left.\chi_{h}, \boldsymbol{\tau}_{h}\right)_{\Omega}-\left(\nabla_{h} w_{h}, \boldsymbol{\tau}_{h}\right)_{\Omega} \\
& -\sum_{e \in \mathcal{E}_{h}^{I}}\left(\mathbf{i} \delta\left\langle\left[\left[\nabla_{h} w_{h}\right]\right],\left[\left[\boldsymbol{\tau}_{h}\right]\right]\right\rangle_{e}-\left\langle\left[\left[w_{h}\right]\right],\left\{\boldsymbol{\tau}_{h}\right\}\right\rangle_{e}\right),
\end{aligned}
$$

we have that $\tilde{\chi}_{h} \in \boldsymbol{\Sigma}_{h}$ satisfies

$$
\begin{aligned}
\left(\tilde{\boldsymbol{\chi}}_{h}, \boldsymbol{\tau}_{h}\right)_{\Omega}= & \left(\nabla_{h} \tilde{w}_{h}, \boldsymbol{\tau}_{h}\right)_{\Omega} \\
& +\sum_{e \in \mathcal{E}_{h}^{I}}\left(\mathbf{i} \delta\left\langle\left[\left[\nabla_{h} \tilde{w}_{h}-\nabla_{h} w\right]\right],\left[\left[\boldsymbol{\tau}_{h}\right]\right]\right\rangle_{e}-\left\langle\left[\left[\tilde{w}_{h}-w\right]\right],\left\{\boldsymbol{\tau}_{h}\right\}\right\rangle_{e}\right) \\
& +\left(\boldsymbol{\chi}, \boldsymbol{\tau}_{h}\right)_{\Omega}-\left(\nabla_{h} w, \boldsymbol{\tau}_{h}\right)_{\Omega} \quad \forall \boldsymbol{\tau}_{h} \in \boldsymbol{\Sigma}_{h} .
\end{aligned}
$$

Lemma 4.1. For any $w, v \in H^{2}\left(\mathcal{T}_{h}\right)$, there exist $k$ - and $h$-independent constants $C$ such that

$$
\left|\mathcal{A}_{h}(w, v)\right| \leq C|||w|\left|\left\|_{1, h}|||v|\right\|_{1, h} .\right.
$$


Moreover, for any $\epsilon \in(0,1)$, there exists a constant $c_{\epsilon}>0$ such that

$$
\operatorname{Re} \mathcal{A}_{h}\left(v_{h}, v_{h}\right)+\left(1-\epsilon+c_{\epsilon}\right) \operatorname{Im} \mathcal{A}_{h}\left(v_{h}, v_{h}\right) \geq(1-\epsilon)\left\|v_{h}\right\|_{1, h}^{2},
$$

where

$$
\begin{aligned}
\|w\|_{1, h}:= & \left(\sum_{K \in \mathcal{T}_{h}}\|\nabla w\|_{L^{2}(K)}^{2}+k\|w\|_{L^{2}(\Gamma)}^{2}\right. \\
& \left.+\sum_{e \in \mathcal{E}_{h}^{I}}\left(\beta\|[w]\|_{L^{2}(e)}^{2}+\delta\left\|\left[\left[\nabla_{h} w\right]\right]\right\|_{L^{2}(e)}^{2}\right)\right)^{\frac{1}{2}}, \\
\||w|\|_{1, h}:= & \left(\|w\|_{1, h}^{2}+\sum_{e \in \mathcal{E}_{h}^{I}} \beta^{-1}\left\|\left\{\nabla_{h} w \cdot \mathbf{n}_{e}\right\}\right\|_{L^{2}(e)}^{2}\right)^{\frac{1}{2}} .
\end{aligned}
$$

Since the proof of the above lemma is elementary, we omit it. We now recall the following stability estimate for $u$ (cf. [7, 10]):

$$
\|u\|_{H^{2}(\Omega)} \lesssim\left(k^{-1}+k\right) M(f, g),
$$

which is needed to prove the next lemma and will be used several times in the rest of this section.

Proposition 4.1. Let $u \in H^{2}(\Omega)$ be the solution to problem (1.1) (1.2) and $\boldsymbol{\sigma}=$ $\nabla u$. Let $\left(\tilde{u}_{h}, \tilde{\boldsymbol{\sigma}}_{h}\right) \in V_{h} \times \boldsymbol{\Sigma}_{h}$ denote the elliptic projection of $(u, \boldsymbol{\sigma})$ defined by (4.1). Then the following error estimates hold:

$$
\begin{aligned}
\left\|u-\tilde{u}_{h}\right\|_{1, h}+k^{\frac{1}{2}}\left\|u-\tilde{u}_{h}\right\|_{L^{2}(\Gamma)} & \lesssim(1+k h)^{\frac{1}{2}} k h, \\
\left\|u-\tilde{u}_{h}\right\|_{L^{2}(\Omega)} & \lesssim(1+k h) k h^{2}, \\
\left\|\boldsymbol{\sigma}-\tilde{\boldsymbol{\sigma}}_{h}\right\|_{L^{2}(\Omega)} & \lesssim(1+k h)^{\frac{1}{2}} k h .
\end{aligned}
$$

Proof. Since the proof of (4.9) and (4.10) is essentially the same as that of 10 , Lemma 5.2], we omit it to save the space and refer the reader to [10 for the details.

To show (4.11), note that (4.4) and the identity $\left(\boldsymbol{\sigma}, \boldsymbol{\tau}_{h}\right)=\left(\nabla_{h} u, \boldsymbol{\tau}_{h}\right)$ imply

$$
\begin{gathered}
\left(\boldsymbol{\sigma}-\tilde{\boldsymbol{\sigma}}_{h}, \boldsymbol{\tau}_{h}\right)_{\Omega}=\left(\nabla_{h} u-\nabla_{h} \tilde{u}_{h}, \boldsymbol{\tau}_{h}\right)_{\Omega}-\sum_{e \in \mathcal{E}_{h}^{I}}\left(\mathbf{i} \delta_{0} h\left\langle\left[\left[\nabla_{h}\left(u-\tilde{u}_{h}\right)\right]\right]\left[\left[\boldsymbol{\tau}_{h}\right]\right]\right\rangle_{e}\right. \\
\left.-\left\langle\left[\left[u-\tilde{u}_{h}\right]\right],\left\{\boldsymbol{\tau}_{h}\right\}\right\rangle_{e}\right) \quad \forall \boldsymbol{\tau}_{h} \in \boldsymbol{\Sigma}_{h}
\end{gathered}
$$


For any $\chi_{h} \in \Sigma_{h}$, we set $\boldsymbol{\tau}_{h}=\chi_{h}-\tilde{\boldsymbol{\sigma}}_{h}$. Then by (4.12), the trace inequality, and the Schwarz inequality, we get

$$
\begin{gathered}
\left\|\boldsymbol{\sigma}-\tilde{\boldsymbol{\sigma}}_{h}\right\|_{L^{2}(\Omega)}^{2}=\left(\boldsymbol{\sigma}-\tilde{\boldsymbol{\sigma}}_{h}, \boldsymbol{\sigma}-\chi_{h}\right)_{\Omega}+\left(\boldsymbol{\sigma}-\tilde{\boldsymbol{\sigma}}_{h}, \boldsymbol{\tau}_{h}\right)_{\Omega} \\
=\left(\boldsymbol{\sigma}-\tilde{\boldsymbol{\sigma}}_{h}, \boldsymbol{\sigma}-\chi_{h}\right)_{\Omega}+\left(\nabla_{h}\left(u-\tilde{u}_{h}\right), \boldsymbol{\tau}_{h}\right)_{\Omega} \\
\quad-\sum_{e \in \mathcal{E}_{h}^{I}}\left(\mathbf{i} \delta_{0} h\left\langle\left[\left[\nabla_{h}\left(u-\tilde{u}_{h}\right)\right]\right],\left[\left[\boldsymbol{\tau}_{h}\right]\right]\right\rangle_{e}-\left\langle\left[\left[u-\tilde{u}_{h}\right]\right],\left\{\boldsymbol{\tau}_{h}\right\}\right\rangle_{e}\right) \\
\leq\left\|\boldsymbol{\sigma}-\tilde{\boldsymbol{\sigma}}_{h}\right\|_{L^{2}(\Omega)}\left\|\boldsymbol{\sigma}-\boldsymbol{\chi}_{h}\right\|_{L^{2}(\Omega)}+\left\|\nabla_{h}\left(u-\tilde{u}_{h}\right)\right\|_{L^{2}(\Omega)}\left\|\boldsymbol{\tau}_{h}\right\|_{L^{2}(\Omega)} \\
\quad+C\left(\sum_{e \in \mathcal{E}_{h}^{I}} \delta_{0} h\left\|\left[\left[\nabla_{h}\left(u-\tilde{u}_{h}\right)\right]\right]\right\|_{L^{2}(e)}^{2}+\beta_{0} h^{-1}\left\|\left[u-\tilde{u}_{h}\right]\right\|_{L^{2}(e)}^{2}\right. \\
\left.\quad+\varepsilon \sum_{e \in \mathcal{E}_{h}^{I}} h\left\|\boldsymbol{\tau}_{h}\right\|_{L^{2}(e)}^{2}\right) \\
\leq \frac{1}{4}\left\|\boldsymbol{\sigma}-\tilde{\boldsymbol{\sigma}}_{h}\right\|_{L^{2}(\Omega)}^{2}+\frac{1}{4}\left\|\boldsymbol{\tau}_{h}\right\|_{L^{2}(\Omega)}^{2}+\left\|\boldsymbol{\sigma}-\boldsymbol{\chi}_{h}\right\|_{L^{2}(\Omega)}^{2}+C\left\|u-\tilde{u}_{h}\right\|_{1, h}^{2} \\
\leq \frac{1}{2}\left\|\boldsymbol{\sigma}-\tilde{\boldsymbol{\sigma}}_{h}\right\|_{L^{2}(\Omega)}^{2}+\frac{5}{4}\left\|\boldsymbol{\sigma}-\chi_{h}\right\|_{L^{2}(\Omega)}^{2}+C\left\|u-\tilde{u}_{h}\right\|_{1, h}^{2} .
\end{gathered}
$$

Hence, it follows from the above inequality, (4.9), and the polynomial approximation theory (cf. [2]) that

$$
\begin{aligned}
\left\|\boldsymbol{\sigma}-\tilde{\boldsymbol{\sigma}}_{h}\right\|_{L^{2}(\Omega)} & \leq C\left\|u-\tilde{u}_{h}\right\|_{1, h}+2 \inf _{\chi_{h} \in \boldsymbol{\Sigma}_{h}}\left\|\boldsymbol{\sigma}-\chi_{h}\right\|_{L^{2}(\Omega)} \\
& \lesssim(1+k h)^{\frac{1}{2}} h k+\left(k+k^{-1}\right) h \\
& \lesssim(1+k h)^{\frac{1}{2}} h k,
\end{aligned}
$$

which gives (4.11). The proof is complete.

4.1.2. Global error estimates for the $L D G$ method \#1. In the preceding subsection we have derived the error bounds for $\left(u-\tilde{u}_{h}, \boldsymbol{\sigma}-\tilde{\boldsymbol{\sigma}}_{h}\right)$. By the decomposition $u-u_{h}=\left(u-\tilde{u}_{h}\right)+\left(\tilde{u}_{h}-u_{h}\right)$ and $\boldsymbol{\sigma}-\boldsymbol{\sigma}_{h}=\left(\boldsymbol{\sigma}-\tilde{\boldsymbol{\sigma}}_{h}\right)+\left(\tilde{\boldsymbol{\sigma}}_{h}-\boldsymbol{\sigma}_{h}\right)$ and the triangle inequality, it suffices to get error bounds for $\left(\tilde{u}_{h}-u_{h}, \tilde{\boldsymbol{\sigma}}_{h}-\boldsymbol{\sigma}_{h}\right)$. We shall accomplish this task by exploiting the linearity of the Helmholtz equation and using the stability estimate for the LDG method \#1 obtained in subsection 3.1 .

First, note that $(u, \boldsymbol{\sigma})$ satisfies

$$
A_{h}\left(u, \boldsymbol{\sigma} ; v_{h}, \boldsymbol{\tau}_{h}\right)=F\left(v_{h}, \boldsymbol{\tau}_{h}\right) \quad \forall\left(v_{h}, \boldsymbol{\tau}_{h}\right) \in V_{h} \times \boldsymbol{\Sigma}_{h} .
$$

Subtracting (3.1) from (4.13) yields the following error equation (or Galerkin orthogonality):

$$
A_{h}\left(u-u_{h}, \boldsymbol{\sigma}-\boldsymbol{\sigma}_{h} ; v_{h}, \boldsymbol{\tau}_{h}\right)=0 \quad \forall\left(v_{h}, \boldsymbol{\tau}_{h}\right) \in V_{h} \times \boldsymbol{\Sigma}_{h} .
$$

Next, to proceed we introduce the notation

$$
\begin{array}{lll}
u-u_{h}=e_{h}+q_{h}, & e_{h}:=u-\tilde{u}_{h}, & q_{h}:=\tilde{u}_{h}-u_{h}, \\
\boldsymbol{\sigma}-\boldsymbol{\sigma}_{h}=\boldsymbol{\psi}_{h}+\phi_{h}, & \boldsymbol{\psi}_{h}:=\boldsymbol{\sigma}-\tilde{\boldsymbol{\sigma}}_{h}, & \phi_{h}:=\tilde{\boldsymbol{\sigma}}_{h}-\boldsymbol{\sigma}_{h} .
\end{array}
$$


Then by (4.14) and the definitions of the sesquilinear form $a_{h}$ and the elliptic projection we have

$$
\begin{aligned}
A_{h}\left(q_{h}, \phi_{h} ; v_{h}, \boldsymbol{\tau}_{h}\right) & =-A_{h}\left(e_{h}, \boldsymbol{\psi}_{h} ; v_{h}, \boldsymbol{\tau}_{h}\right) \\
& =-a_{h}\left(e_{h}, \boldsymbol{\psi}_{h} ; v_{h}, \boldsymbol{\tau}_{h}\right)+k^{2}\left(e_{h}, v_{h}\right) \\
& =k^{2}\left(e_{h}, v_{h}\right), \quad \forall\left(v_{h}, \boldsymbol{\tau}_{h}\right) \in V_{h} \times \boldsymbol{\Sigma}_{h} .
\end{aligned}
$$

The above equation implies that $\left(q_{h}, \boldsymbol{\phi}_{h}\right) \in V_{h} \times \boldsymbol{\Sigma}_{h}$ is the LDG solution to the Helmholtz problem with source terms $f=k^{2} e_{h}$ and $g=0$. Then an application of the stability estimates of Theorem 3.1 immediately yields the following lemma.

Proposition 4.2. The following estimates hold for $\left(q_{h}, \phi_{h}\right)$ :

$$
\begin{aligned}
\left\|q_{h}\right\|_{D G} & \lesssim \gamma_{1}(1+k h) k^{2} h^{2} \\
\left\|\phi_{h}\right\|_{L^{2}(\Omega)} & \lesssim \gamma_{1}\left(1+k h+\delta_{0} k h^{2}\right)(1+k h) k h .
\end{aligned}
$$

Combining Propositions 4.1 and 4.2 using the triangle inequality and the standard duality argument give the following error estimates for $\left(u_{h}, \boldsymbol{\sigma}_{h}\right)$.

Theorem 4.1. Let $u \in H^{2}(\Omega)$ be the solution to problem (1.1) -(1.2) and $\boldsymbol{\sigma}:=\nabla u$, and let $\left(u_{h}, \boldsymbol{\sigma}_{h}\right)$ be the solution to problem (3.1). Then the following error estimates for $\left(u_{h}, \boldsymbol{\sigma}_{h}\right)$ hold:

$$
\begin{aligned}
& \left\|u-u_{h}\right\|_{1, h}+k^{\frac{1}{2}}\left\|u-u_{h}\right\|_{L^{2}(\Gamma)} \lesssim\left((1+k h)^{\frac{1}{2}}+\gamma_{1}(1+k h) k h\right) k h, \\
& \left\|u-u_{h}\right\|_{L^{2}(\Omega)} \lesssim\left(1+\gamma_{1}\right)(1+k h) k h^{2}, \\
& \left\|\boldsymbol{\sigma}-\boldsymbol{\sigma}_{h}\right\|_{L^{2}(\Omega)} \lesssim\left((1+k h)^{\frac{1}{2}}+\gamma_{1}(1+k h)\left(1+k h+\delta_{0} k h^{2}\right)\right) k h .
\end{aligned}
$$

Remark 4.1. Substituting the expression of $\gamma_{1}$ (see (3.5) into (4.18), we obtain the following error bound which shows the explicit dependence on $h$ and $k$ :

$$
\left\|u-u_{h}\right\|_{1, h} \lesssim\left((1+k h)^{\frac{1}{2}}+\left(1+(k h)^{-1}+(k h)^{-2}\right)(1+k h) k h\right) k h .
$$

In particular, if $k^{3} h^{2} \lesssim 1$, we have

$$
\left\|u-u_{h}\right\|_{1, h} \lesssim k h+k^{3} h^{2}
$$

The above error bound has the same asymptotic order (in both $h$ and $k$ ) as that for the one-dimensional finite element method (cf. [17]) as well as that for the IPDG method (cf. [10]). We note that our numerical tests in the next section (see Figure 5) suggest that $\left\|u-u_{h}\right\|_{1, h}$ decreases in $h$ when $k^{3} h^{2}=1$ holds.

4.2. Error estimates for the LDG method \#2. The error analysis for the LDG method \#2 essentially follows the same lines as that for the LDG method \#1 given in the previous subsection. However, there are three main differences which we now explain. First, the sesquilinear form $a_{h}$ needs to be replaced by another sesquilinear form $b_{h}$ in the definition of the elliptic projection (4.1), where $b_{h}$ is 
defined by

$$
\begin{aligned}
b_{h}\left(w_{h}, \chi_{h} ; v_{h}, \boldsymbol{\tau}_{h}\right):= & B_{h}\left(w_{h}, \chi_{h} ; v_{h}, \boldsymbol{\tau}_{h}\right)+k^{2}\left(w_{h}, v_{h}\right)_{\Omega} \\
= & \left(\boldsymbol{\chi}_{h}, \nabla_{h} v_{h}\right)_{\Omega}+\mathbf{i} k\left\langle w_{h}, v_{h}\right\rangle_{\Gamma} \\
& -\sum_{e \in \mathcal{E}_{h}^{I}}\left\langle\left\{\chi_{h}\right\}-\mathbf{i} \beta\left[\left[w_{h}\right]\right],\left[\left[v_{h}\right]\right]\right\rangle_{e} \\
& -\sum_{e \in \mathcal{E}_{h}^{I}}\left(\mathbf{i} \delta\left\langle\left[\left[\boldsymbol{\chi}_{h}\right]\right],\left[\left[\boldsymbol{\tau}_{h}\right]\right]\right\rangle_{e}-\left\langle\left[\left[w_{h}\right]\right],\left\{\boldsymbol{\tau}_{h}\right\}\right\rangle_{e}\right) \\
& +\left(\boldsymbol{\chi}_{h}, \boldsymbol{\tau}_{h}\right)_{\Omega}-\left(\nabla_{h} w_{h}, \boldsymbol{\tau}_{h}\right)_{\Omega},
\end{aligned}
$$

and is a coercive bilinear form.

Second, due to strong coupling between $\tilde{u}_{h}$ and $\tilde{\boldsymbol{\sigma}}_{h}$, the error estimates for the new elliptic projection $\left(\tilde{u}_{h}, \tilde{\boldsymbol{\sigma}}_{h}\right)$ must be derived differently. To this end, we need the following lemma, which replaces Lemma 4.1 .

Lemma 4.2. Let $\beta=\beta_{0} h^{-1}$ and $\delta=\delta_{0} h$ for some positive constants $\beta_{0}$ and $\delta_{0}$.

(i) There exist $h$ - and $k$-independent constants $c_{3}>0$ such that the sesquilinear form $b_{h}$ satisfies the following generalized inf-sup condition: for any fixed $\left(w_{h}, \chi_{h}\right) \in$ $V_{h} \times \Sigma_{h}$,

$$
\begin{aligned}
\sup _{\left(v_{h}, \boldsymbol{\tau}_{h}\right) \in V_{h} \times \boldsymbol{\Sigma}_{h}} & \frac{\operatorname{Re} b_{h}\left(w_{h}, \boldsymbol{\chi}_{h} ; v_{h}, \boldsymbol{\tau}_{h}\right)}{\left\|\left|\left(v_{h}, \boldsymbol{\tau}_{h}\right)\right|\right\| \|_{D G}} \\
& +\sup _{\left(v_{h}, \boldsymbol{\tau}_{h}\right) \in V_{h} \times \boldsymbol{\Sigma}_{h}} \frac{\operatorname{Im} b_{h}\left(w_{h}, \boldsymbol{\chi}_{h} ; v_{h}, \boldsymbol{\tau}_{h}\right)}{\left\|\mid\left(v_{h}, \boldsymbol{\tau}_{h}\right)\right\| \|_{D G}} \geq c_{3}\left\|\left|\left(w_{h}, \boldsymbol{\chi}_{h}\right)\right|\right\|_{D G} .
\end{aligned}
$$

(ii) There exist $h$ - and $k$-independent constants $C>0$ such that for any $(w, \chi)$, $(v, \boldsymbol{\tau}) \in H^{2}\left(\mathcal{T}_{h}\right) \times H^{1}\left(\mathcal{T}_{h}\right)^{d}$, it holds that

$$
\left|b_{h}(w, \chi ; v, \boldsymbol{\tau})\right| \leq C|||(w, \chi)|||_{1, h}|||(v, \boldsymbol{\tau})| \|_{1, h},
$$

where

$$
\begin{aligned}
\|(w, \chi)\| \|_{D G} & :=\left(\|w\|_{1, h}^{2}+\|\chi\|_{L^{2}(\Omega)}^{2}\right)^{\frac{1}{2}} \\
\|(w, \chi) \mid\|_{1, h} & :=\left(\|(w, \chi) \mid\|_{D G}^{2}+\sum_{e \in \mathcal{E}_{h}^{I}} \beta^{-1}\|\{\chi\}\|_{L^{2}(e)}^{2}\right)^{\frac{1}{2}} .
\end{aligned}
$$

The proof of (i) is based on evaluating the first quotient on the left-hand side of (4.22) at $\left(v_{h}, \boldsymbol{\tau}_{h}\right)=\left(\left(1+C_{1}\right) w_{h}, C_{1} \chi_{h}-\nabla_{h} w_{h}\right)$ and evaluating the second quotient at $\left(v_{h}, \boldsymbol{\tau}_{h}\right)=\left(C_{2} w_{h}, C_{2} \chi_{h}\right)$ for some sufficiently large positive constants $C_{1}$ and $C_{2}$. The proof of (ii) is a straightforward application of Schwarz and trace inequalities. We skip the rest of the derivation to save space.

The above generalized inf-sup condition, the boundedness of the sesquilinear form $b_{h}$, and the duality argument (cf. 2] ) readily infer the following error estimates for the new elliptic projection $\left(\tilde{u}_{h}, \tilde{\boldsymbol{\sigma}}_{h}\right)$. We omit the proof since it is standard.

Proposition 4.3. Under the assumptions of Proposition 4.1, the following estimates hold:

$$
\begin{aligned}
\left\|u-\tilde{u}_{h}\right\|_{1, h}+\left\|\boldsymbol{\sigma}-\tilde{\boldsymbol{\sigma}}_{h}\right\|_{L^{2}(\Omega)} & \lesssim k h, \\
\left\|u-\tilde{u}_{h}\right\|_{L^{2}(\Omega)} & \lesssim k h^{2} .
\end{aligned}
$$


The third difference is that the new error function $\left(q_{h}, \phi_{h}\right)$ now satisfies

$$
B_{h}\left(q_{h}, \phi_{h} ; v_{h}, \boldsymbol{\tau}_{h}\right)=k^{2}\left(e_{h}, v_{h}\right) \quad \forall\left(v_{h}, \boldsymbol{\tau}_{h}\right) \in V_{h} \times \boldsymbol{\Sigma}_{h} .
$$

As a result, by Theorem 3.3 and (3.21) we get

$$
\left|q_{h}\right|_{1, h}+\left\|\left(q_{h}, \phi_{h}\right)\right\|_{D G} \lesssim \gamma_{2}(1+k h) k^{2} h^{2},
$$

which replaces estimates (4.16) and (4.17).

After having established Proposition 4.3 and (4.29), once again, by the triangle inequality, we arrive at the following error estimates for the solution $\left(u_{h}, \boldsymbol{\sigma}_{h}\right)$ to the LDG method \#2.

Theorem 4.2. Let $u \in H^{2}(\Omega)$ be the solution to problem (1.1) (1.2) and $\boldsymbol{\sigma}:=$ $\nabla u$, and let $\left(u_{h}, \boldsymbol{\sigma}_{h}\right)$ be the solution to problem (3.17). Then the following error estimates hold for $\left(u_{h}, \boldsymbol{\sigma}_{h}\right)$ :

$$
\begin{aligned}
\left\|u-u_{h}\right\|_{1, h}+ & k^{\frac{1}{2}}\left\|u-u_{h}\right\|_{L^{2}(\Gamma)} \\
& \left.+\left\|\boldsymbol{\sigma}-\boldsymbol{\sigma}_{h}\right\|_{L^{2}(\Omega)} \lesssim\left(1+\gamma_{2}(1+k h) k h\right)\right) k h, \\
\left\|u-u_{h}\right\|_{L^{2}(\Omega)} & \lesssim\left(1+\gamma_{2}(1+k h)\right) k h^{2} .
\end{aligned}
$$

Remark 4.2. (4.29) shows that $\phi_{h}:=\tilde{\boldsymbol{\sigma}}_{h}-\boldsymbol{\sigma}_{h}$ has an optimal order (in $h$ ) error bound for the LDG method \#2, while (4.17) shows that $\phi_{h}$ only has a sub-optimal order error bound for the LDG method \#1. We believe that this is the main reason why in practice the LDG method \#2 gives a better approximation to the flux variable $\boldsymbol{\sigma}$ than the LDG method \#1 does, although both methods have the same asymptotic rate of convergence in $h$.

\section{NUMERICAL EXPERIMENTS}

In this section we shall provide some numerical results of the two proposed LDG methods. Our tests are done for the following two-dimensional Helmholtz problem:

$$
\begin{aligned}
-\Delta u-k^{2} u & =f:=\frac{\sin (k r)}{r} & & \text { in } \Omega, \\
\frac{\partial u}{\partial n_{\Omega}}+\mathrm{i} k u & =g & & \text { on } \Gamma_{R}:=\partial \Omega .
\end{aligned}
$$

Here $\Omega$ is the unit square $[-0.5,0.5] \times[-0.5,0.5]$, and $g$ is chosen so that the exact solution is given by

$$
u=\frac{\cos (k r)}{k}-\frac{\cos k+\mathbf{i} \sin k}{k\left(J_{0}(k)+\mathbf{i} J_{1}(k)\right)} J_{0}(k r)
$$

in polar coordinates, where $J_{\nu}(z)$ are Bessel functions of the first kind.

Assume $\mathcal{T}_{1 / m}$ be the regular triangulation that consists of $2 m^{2}$ right-angled equicrural triangles of size $h=1 / m$, for any positive integer $m$. See Figure 1 for the sample triangulation $\mathcal{T}_{1 / 4}$ and $\mathcal{T}_{1 / 10}$.

5.1. Sensitivity with respect to the parameters $\delta$ and $\beta$. In this subsection, we examine the sensitivity of the error of the LDG solutions in $H^{1}$-seminorm with respect to the parameters $\delta$ and $\beta$.

The LDG method \#1 is considered first. We start by fixing $\delta=0.1 h_{e}$ and testing the sensitivity in the parameter $\beta$. With two wave numbers $k=5$ and 50 , we compute the solutions of the LDG method \#1 with different values of $\beta$ : $0.001 h_{e}^{-1}, 0.01 h_{e}^{-1}, h_{e}^{-1}$ and 1 . The relative errors, defined by the errors in the 

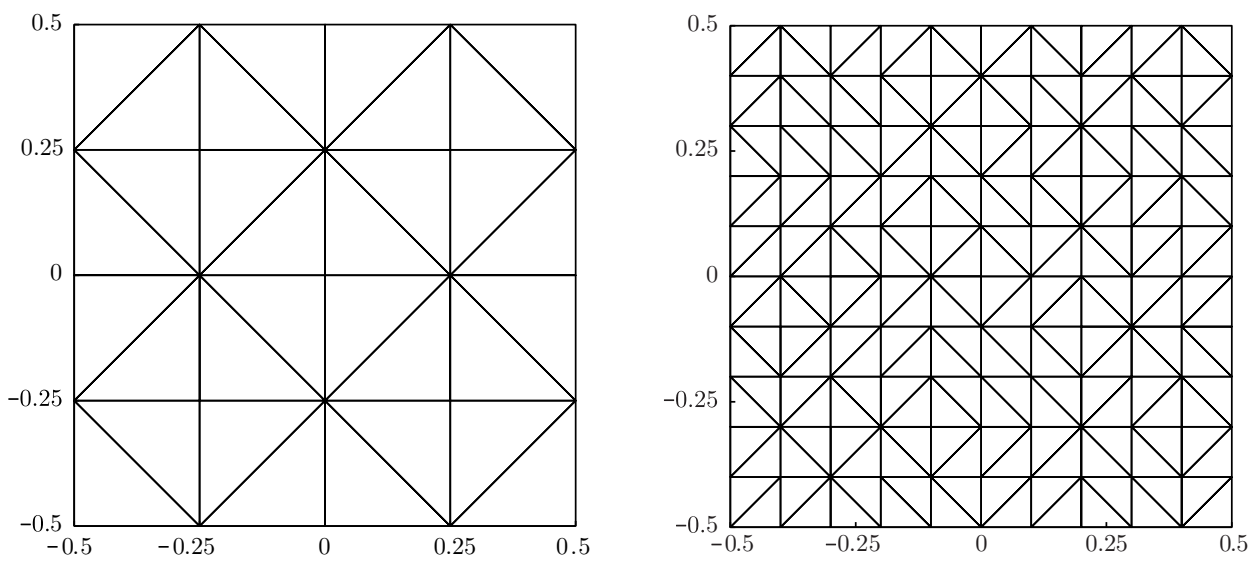

Figure 1. The computational domain and sample meshes. Left: $\mathcal{T}_{1 / 4}$ that consists of right-angled equicrural triangles of size $h=\frac{1}{4}$; Right: $\mathcal{T}_{1 / 10}$ with $h=\frac{1}{10}$.

$H^{1}$-seminorm divided by the exact solution in the $H^{1}$-seminorm, are shown in the left graph of Figure 2. We observe that the relative errors have similar behaviors and decay as mesh size $h$ becomes smaller. This shows that the errors are not sensitive to the parameter $\beta$. Next, we fix $\beta=0.001 h_{e}^{-1}$, and repeat the test with different $\delta$. The right graph of Figure 2 shows the relative errors with parameters $\delta=0.001 h_{e}, 0.1 h_{e}, 10 h_{e}$ and 0.1 , and wave numbers $k=5$ and 50 . We observe that the errors have similar behaviors for small values $\delta=0.001 h_{e}$ and $0.1 h_{e}$. Larger $\delta$ results in larger error.

The sensitivity tests of the LDG method \#2 are shown in Figure 3. similar behaviors are also observed.
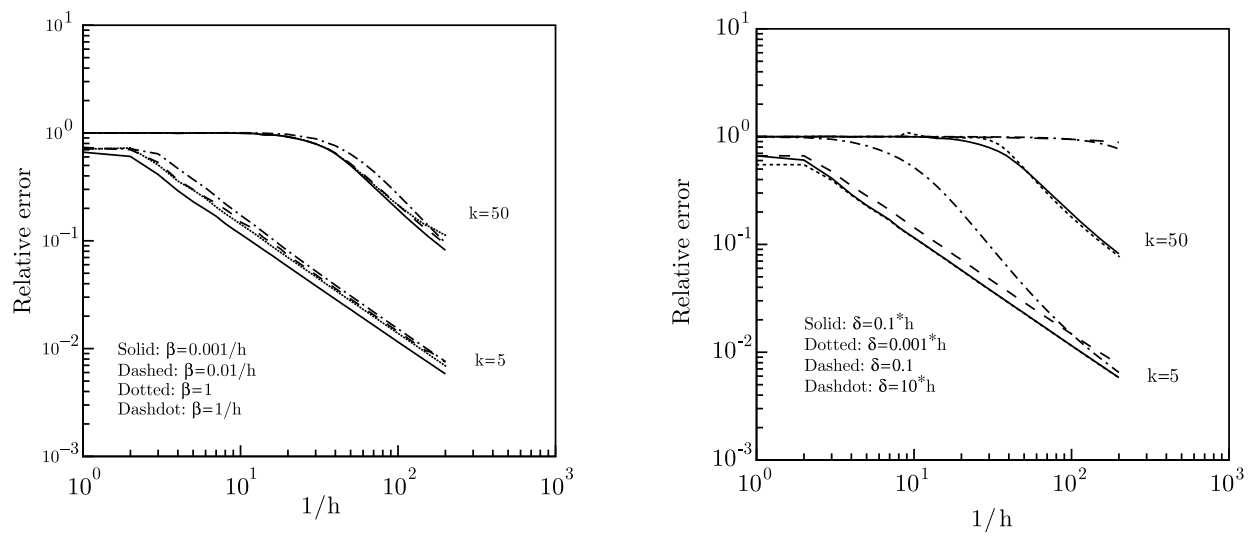

Figure 2. Relative error in the $H^{1}$-seminorm of the LDG method \#1 with different parameters for two wave numbers $k=5$ and 50 . Left: $\delta=0.1 h_{e}$ is fixed, $\beta=0.001 h_{e}^{-1}, 0.01 h_{e}^{-1}, h_{e}^{-1}$ and 1; Right: $\beta=0.001 h_{e}^{-1}$ is fixed, $\delta=0.001 h_{e}, 0.1 h_{e}, 10 h_{e}$ and 0.1 . 

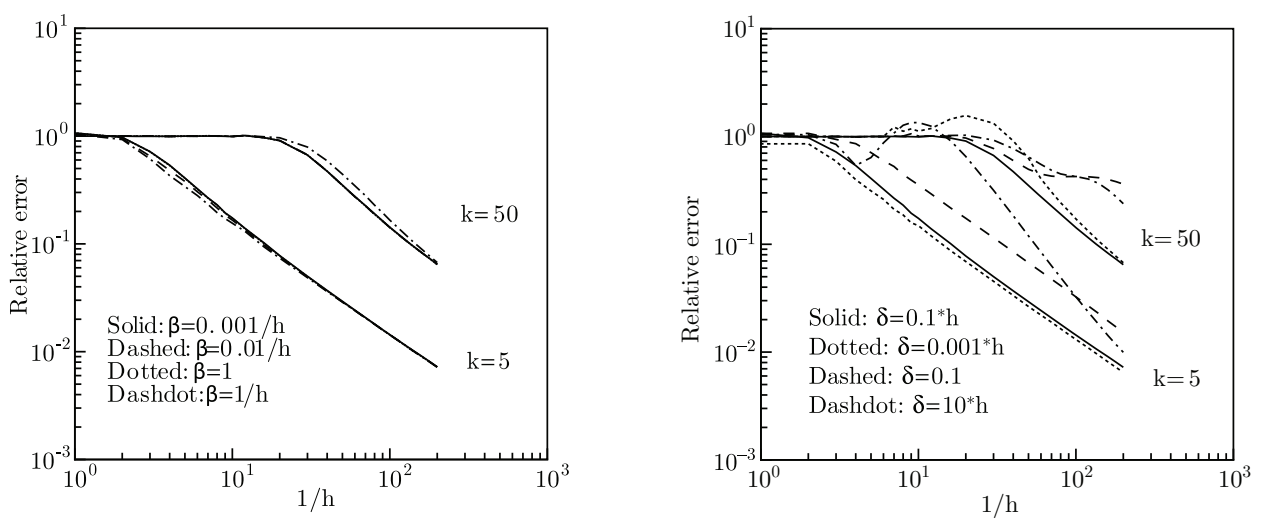

Figure 3. Relative error in the $H^{1}$-seminorm of the LDG method \#2 with different parameters for two wave numbers $k=5$ and 50 . Left: $\delta=0.1 h_{e}$ is fixed, $\beta=0.001 h_{e}^{-1}, 0.01 h_{e}^{-1}, h_{e}^{-1}$ and 1; Right: $\beta=0.001 h_{e}^{-1}$ is fixed, $\delta=0.001 h_{e}, 0.1 h_{e}, 10 h_{e}$ and 0.1 .

5.2. Errors of the LDG solutions. In this subsection, we fix the parameters and investigate the changes of the numerical errors as functions of the mesh size.

We start from the LDG method \#1. As suggested by the sensitivity tests in the previous subsection, we pick

$$
\delta=0.1 h_{e}, \quad \beta=0.001 h_{e}^{-1} .
$$

The relative error of the LDG method, and the finite element interpolation are shown in the left graph of Figure 4, with four different wave numbers $k=5,10$, 50 and 100. The relative error of the LDG solution stays around $100 \%$ before a critical mesh size is reached, then decays at a rate greater than -1 in the log-log scale but converges as fast as the finite element interpolation (with slope -1 ) for small $h$. The critical mesh size decreases as $k$ increases.

The right graph of Figure 4 contains the relative error when we fix $k h=1$ and $h k=0.5$. It indicates that unlike the error of the finite element interpolation the error of the LDG is not controlled by the magnitude of $k h$, which suggests that there is a pollution contribution in the total error. The left graph of Figure 5 contains the relative error of the LDG method with the mesh size satisfying $k^{3} h^{2}=1$ for different values of $h$. The error does not increase with respect to $k$.

The LDG method \#2 has also been tested using the same parameters in (5.4). Similar behaviors have been observed as shown in Figure 5 and 6 .

At the end, we look closely at the situation with a large relative error when $k h>1$. The LDG method \#1 solution with parameters $\delta=0.1 h_{e}, \beta=0.001 h_{e}^{-1}$, $k=100$ and $h=1 / 60$ has a large relative error of size 0.9392 . The surface plots of the finite element interpolation and the LDG solution are given in Figure 7 . It shows that the LDG solution has the correct shape/phase although its amplitude is smaller. 

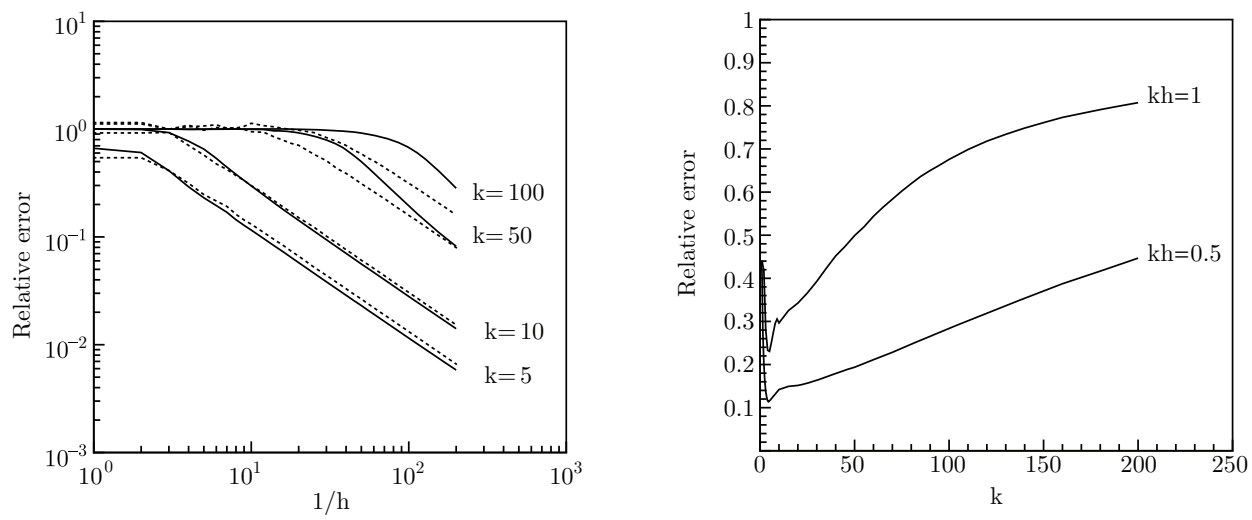

FiguRE 4. Left: relative error of the LDG method \#1 (solid line) and the finite element interpolation (dotted line) in the $H^{1}$ seminorm for $k=5,10,50$ and 100; Right: relative error of the LDG method \#1 in the $H^{1}$-seminorm for $k=1, \ldots, 200, k h=1$ and $k h=0.5$.
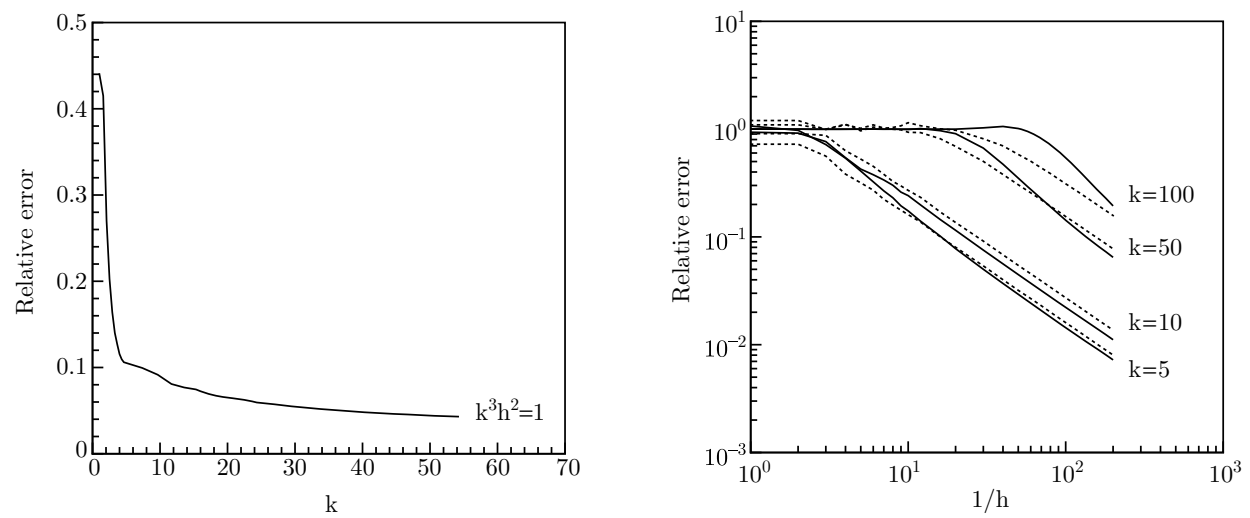

FIGURE 5. Left: relative error of the LDG method \#1 in the $H^{1}$ seminorm with $k^{3} h^{2}=1$; Right: relative error of the LDG method \#2 (solid line) and the finite element interpolation (dotted line) in the $H^{1}$-seminorm for $k=5,10,50$ and 100 .

5.3. Comparison between the two LDG methods. Two different LDG methods are proposed in this paper. The first one is derived following the IPDG method proposed in [10, and the second one has a more standard numerical flux formulation and is supposed to have a better approximation for the vector/flux variable. In this subsection, we provide a comparison between these two methods, in terms of the error and computational cost. 

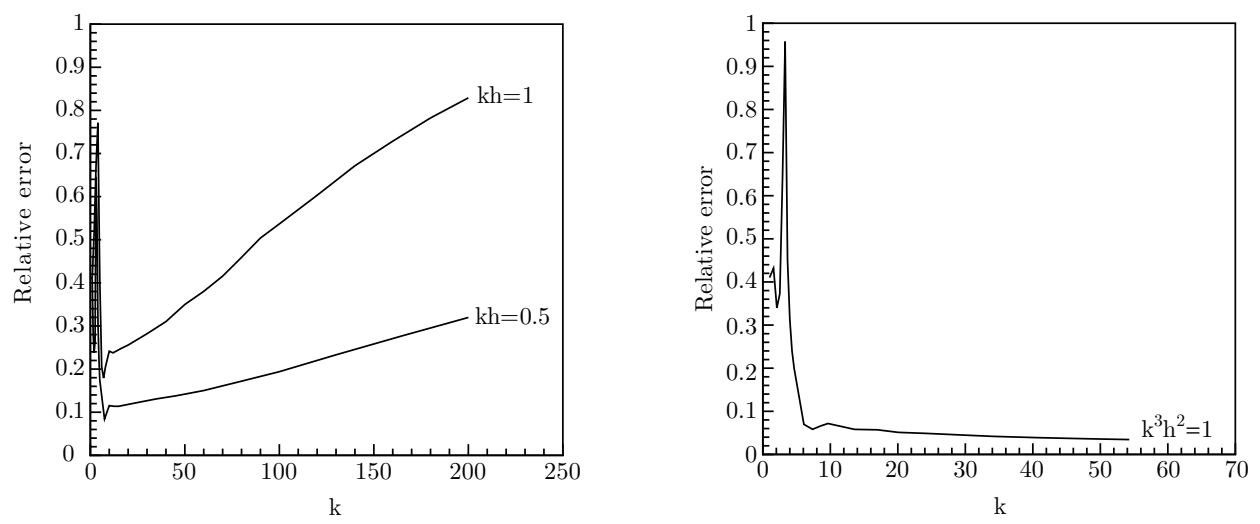

Figure 6. Relative error of the LDG method \#2 in the $H^{1}$ seminorm. Left: $k h=1$ and $k h=0.5$ Right: $k^{3} h^{2}=1$.
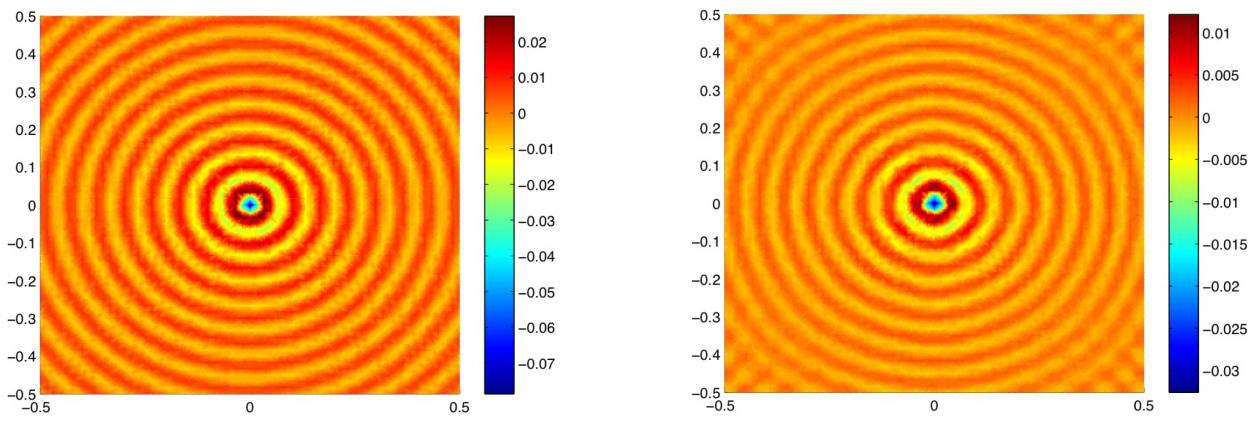

FiguRE 7. Left: surface plots of the finite element interpolation (left) and the LDG method \#1 solution (right) with parameters $\delta=0.1 h_{e}, \beta=0.001 h_{e}^{-1}, k=100$ and $h=1 / 60$.

We start by revisiting the test examples of subsection 5.1 Instead of computing the relative error of $u_{h}$ in the $H^{1}$-seminorm, we compute the relative error of $\boldsymbol{\sigma}_{h}$ in the $L^{2}$-norm for the LDG method \#2. The numerical results are presented in Figure 8 which show that although the solution is still not sensitive to the parameter $\beta$, better approximation to $\sigma$ is achieved for larger $\delta$. It confirms our prediction that the LDG method \#2 gives a better approximation for the vector/flux variable.

Table 1 provides a detailed comparison of these two methods for different mesh sizes $h$, with the parameters $\delta=0.1 h_{e}, \beta=0.001 h_{e}^{-1}$ and $k=10$. It shows that the computational cost of the LDG method \#2 is about twice the size of the LDG method \#1. Also, as expected, the error of the vector/flux variable of the LDG method \#2 is smaller than that of the LDG method \#1, and both methods demonstrate a first order rate of convergence. 

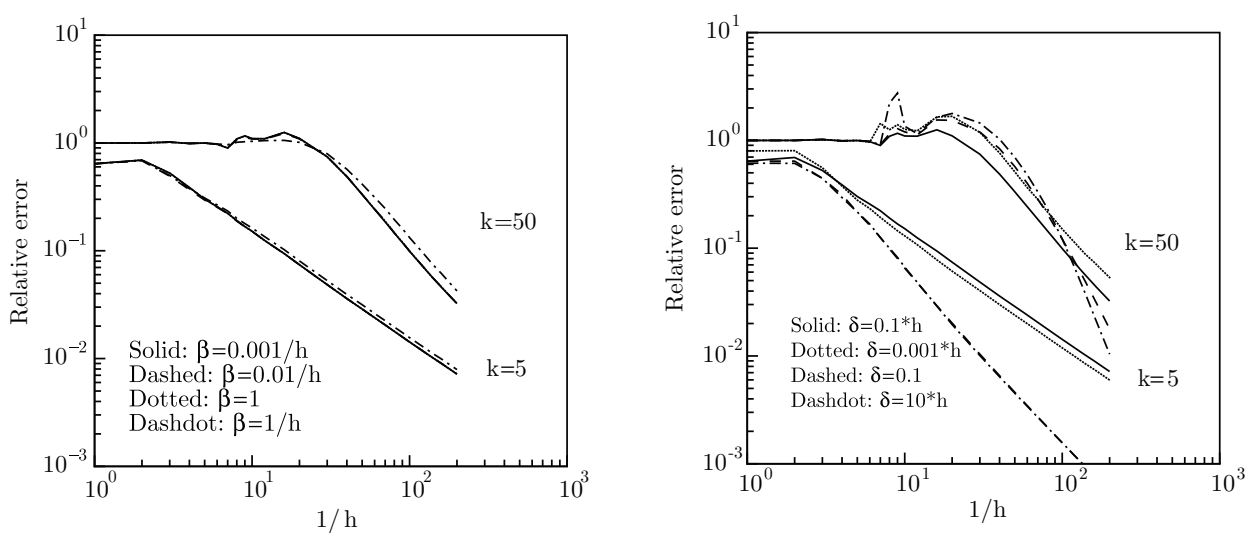

FIGURE 8. Relative error of $\boldsymbol{\sigma}_{h}$ in $L^{2}$-norm of the LDG method \#2 with different parameters for two wave numbers $k=5$ and 50 . Left: $\delta=0.1 h_{e}$ is fixed, $\beta=0.001 h_{e}^{-1}, 0.01 h_{e}^{-1}, h_{e}^{-1}$ and 1; Right: $\beta=0.001 h_{e}^{-1}$ is fixed, $\delta=0.001 h_{e}, 0.1 h_{e}, 10 h_{e}$ and 0.1 .

TABLE 1. Comparison of the two LDG methods with parameters $\delta=0.1 h_{e}$ and $\beta=0.001 h_{e}^{-1}$.

\begin{tabular}{|c|c|c|c|c|c|c|}
\hline & $1 / h$ & $\left|u-u_{h}\right|_{H^{1}}$ & order & $\left\|\boldsymbol{\sigma}-\boldsymbol{\sigma}_{h}\right\|_{L^{2}}$ & order & CPU time (s) \\
\hline \multirow{5}{*}{ LDG \#1 } & 5 & $4.1059 \mathrm{E}-01$ & & $5.4715 \mathrm{E}-01$ & & 0.0641 \\
\cline { 2 - 7 } & 10 & $1.6915 \mathrm{E}-01$ & 1.2794 & $2.4712 \mathrm{E}-01$ & 1.1467 & 0.2381 \\
\cline { 2 - 7 } & 20 & $7.6089 \mathrm{E}-02$ & 1.1525 & $1.1804 \mathrm{E}-01$ & 1.0659 & 0.9671 \\
\cline { 2 - 7 } & 40 & $3.6648 \mathrm{E}-02$ & 1.0539 & $5.7114 \mathrm{E}-02$ & 1.0474 & 3.9380 \\
\cline { 2 - 7 } & 80 & $1.8151 \mathrm{E}-02$ & 1.0137 & $2.8319 \mathrm{E}-02$ & 1.0121 & 15.8194 \\
\cline { 2 - 7 } & 160 & $9.0379 \mathrm{E}-03$ & 1.0060 & $1.4004 \mathrm{E}-02$ & 1.0159 & 69.1861 \\
\hline \hline \multirow{5}{*}{ LDG $\# 2$} & 5 & $2.4711 \mathrm{E}-01$ & & $2.2184 \mathrm{E}-01$ & & 0.1057 \\
\cline { 2 - 7 } & 10 & $1.4040 \mathrm{E}-01$ & 0.8156 & $7.6775 \mathrm{E}-02$ & 1.5308 & 0.4368 \\
\cline { 2 - 7 } & 20 & $6.6992 \mathrm{E}-02$ & 1.0675 & $3.3630 \mathrm{E}-02$ & 1.1909 & 1.8356 \\
\cline { 2 - 7 } & 40 & $3.2693 \mathrm{E}-02$ & 1.0350 & $1.5710 \mathrm{E}-02$ & 1.0981 & 7.9357 \\
\cline { 2 - 7 } & 80 & $1.6165 \mathrm{E}-02$ & 1.0161 & $7.7418 \mathrm{E}-03$ & 1.0209 & 34.5688 \\
\cline { 2 - 7 } & 160 & $8.0949 \mathrm{E}-03$ & 0.9978 & $3.9127 \mathrm{E}-03$ & 0.9845 & 157.6018 \\
\hline
\end{tabular}

5.4. Comparison between LDG and finite element solutions. We have shown the performance and comparison of the two LDG methods in previous subsections. In this subsection, we provide a brief comparison between the LDG solution and the $P_{1}$ conforming finite element solution.

We consider the Helmholtz problem (5.1) -(5.2) with wave number $k=100$. With mesh size $h=1 / 50,1 / 120$ and $1 / 200$, we plot the traces of the LDG method \#1 solution with parameters (5.4) in $x z$-plane in the left column of Figure 9 . The exact solution is also provided as a reference. The traces of the finite element solution are shown in the right column of Figure 9. It is clear that the LDG method \#1 has a better approximation to the exact solution. Larger phase error in the finite element solution is observed in all three cases. Also, the LDG solution has a better approximation for the amplitude of the exact solution. 

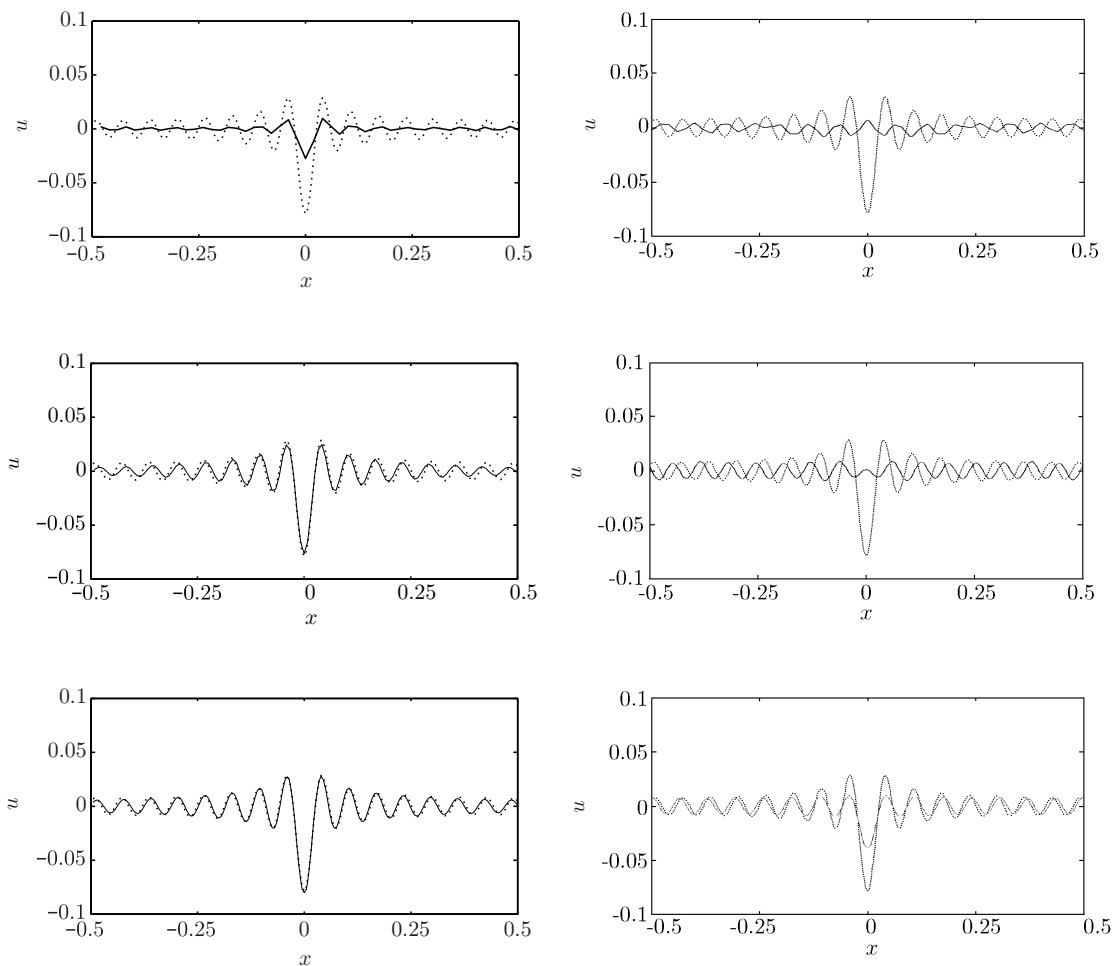

Figure 9. The traces of the LDG \#1 solution (left) and the finite element solution (right) in the $x z$-plane, for $k=100$ and $h=$ $1 / 50$ (top), 1/120 (middle) and 1/200 (bottom), respectively. The dotted lines are the traces of the exact solution.

\section{ACKNOWLEDGMENTS}

The authors would like to thank Professor Haijun Wu of Nanjing University of China for a helpful discussion which led to the simplified proofs of Propositions 3.1 and 3.2 compared to those given in an earlier version of the paper [12. The authors would also like to thank Professor Bernardo Cockburn of the University of Minnesota for his helpful discussions.

\section{REFERENCES}

[1] D. Arnold, F. Brezzi, B. Cockburn, and D. Marini. Unified analysis of discontinuous Galerkin methods for elliptic problems. SIAM J. Numer. Anal., 39:1749-1779, 2002. MR 1885715 (2002k:65183)

[2] S. C. Brenner and L. R. Scott. The mathematical theory of finite element methods. 3rd ed. Texts in Applied Mathematics, vol. 15, Springer, New York, 2008. MR2373954|(2008m:65001)

[3] P. Castillo, B. Cockburn, I. Perugia and D. Schötzau. Local discontinuous Galerkin method for elliptic problems. Commun. Numer. Meth. Engrg., 18:69-75, 2002.

[4] B. Cockburn, J. Gopalakrishnan, and R. Lazarov. Unified hybridization of discontinuous Galerkin, mixed, and continuous Galerkin methods for second order elliptic problems. SIAM J. Numer. Anal., 47(2):1319-1365, 2009. MR2485455(2010b:65251) 
[5] B. Cockburn, G. E. Karniadakis, C.-W. Shu. Discontinuous Galerkin Methods, Theory, Computation, and Applications. Springer Lecture Notes in Computational Science and Engineering, vol. 11, Springer-Verlag, 2000. MR 1842160 (2002b:65004)

[6] B. Cockburn and C. -W. Shu. The local discontinuous Galerkin method for convectiondiffusion systems. SIAM J. Numer. Anal., 35:2440-2463, 1998. MR.1655854 (99j:65163)

[7] P. Cummings and X. Feng. Sharp regularity coefficient estimates for complex-valued acoustic and elastic Helmholtz equations. Math. Models Methods Appl. Sci., 16:139-160, 2006. MR2194984(2007d:35030)

[8] J. Douglas, Jr., J. E. Santos, D. Sheen, and L. S. Bennethum. Frequency domain treatment of one-dimensional scalar waves. Math. Models Methods Appl. Sci., 3(2):171-194, 1993. MR:1212938(94g:65111)

[9] B. Engquist and A. Majda. Radiation boundary conditions for acoustic and elastic wave calculations. Comm. Pure Appl. Math., 32(3):314-358, 1979. MR517938 (80e:76041)

[10] X. Feng and H. Wu. Discontinuous Galerkin methods for the Helmholtz equation with large wave numbers. SIAM J. Numer. Anal., 47:2872-2896, 2009. MR2551150(2011a:65399)

[11] X. Feng and H. Wu. hp-discontinuous Galerkin methods for the Helmholtz equation with large wave numbers. Math. Comp., 80:1997-2024, 2011. MR2813347

[12] X. Feng and Y. Xing. Absolutely stable local discontinuous Galerkin methods for the Helmholtz equation with large wave number. arXiv:1010.4563v1 [math.NA].

[13] R. Griesmaier and P. Monk. Error analysis for a hybridizable discontinuous Galerkin method for the Helmholtz equation. J. Scient. Computing, 49:291-310, 2011.

[14] U. Hetmaniuk. Stability estimates for a class of Helmholtz problems. Commun. Math. Sci., 5(3):665-678, 2007. MR 2352336(2008m:35050)

[15] R. Hiptmair and I. Perugia. Mixed plane wave discontinuous Galerkin methods. Lecture Notes in Computational Science and Engineering, 70:51-62, 2009. MR2743958

[16] F. Ihlenburg. Finite Element Analysis of Acoustic Scattering. Springer-Verlag, New York, 1998. MR $1639879(99 \mathrm{~g}: 65114)$

[17] F. Ihlenburg and I. Babuška. Finite element solution of the Helmholtz equation with high wave number. I. The $h$-version of the FEM. Comput. Math. Appl., 30(9):9-37, 1995. MR1353516 (96j:65123)

[18] T. Luostari, T. Huttunen, and P. Monk. Plane wave methods for approximating the time harmonic wave equation. Highly oscillatory problems. London Math. Soc. Lecture Notes, 366:127-153, 2009. MR2562508 (2010k:65262)

[19] B. Rivière. Discontinuous Galerkin methods for solving elliptic and parabolic equations. SIAM, Philadelphia, PA, 2008. MR2431403(2009f:65260)

[20] O. C. Zienkiewicz. Achievements and some unsolved problems of the finite element method. Internat. J. Numer. Methods Engrg, 47:9-28, 2000. MR1744287 (2000m:65002)

Department of Mathematics, The University of Tennessee, Knoxville, Tennessee 37996

E-mail address: xfeng@math.utk.edu

Department of Mathematics, The University of Tennessee, Knoxville, Tennessee 37996 - And - Computer Science and Mathematics Division, Oak Ridge National Laboratory, OAK Ridge, Tennessee 37830

E-mail address: xingy@math.utk.edu 